\title{
Special weak Dirichlet processes and BSDEs driven by a random measure
}

\author{
ELENA BANDINI ${ }^{1}$ and FRANCESCO RUSSO${ }^{2}$ \\ ${ }^{1}$ LUISS Roma, Department of Economics and Finance, Viale Romania 32, 00197 Roma, Italy. \\ E-mail:ebandini@luiss.it \\ ${ }^{2}$ ENSTA ParisTech, Université Paris-Saclay, Unité de Mathématiques appliquées, 828, Boulevard des \\ Maréchaux, 91120 Palaiseau, France. E-mail: francesco.russo@ensta-paristech.fr
}

This paper considers a forward BSDE driven by a random measure, when the underlying forward process $X$ is a special semimartingale, or even more generally, a special weak Dirichlet process. Given a solution $(Y, Z, U)$, generally $Y$ appears to be of the type $u\left(t, X_{t}\right)$ where $u$ is a deterministic function. In this paper, we identify $Z$ and $U$ in terms of $u$ applying stochastic calculus with respect to weak Dirichlet processes.

Keywords: backward stochastic differential equations; random measure; stochastic integrals for jump processes; weak Dirichlet processes

\section{Introduction}

This paper considers a forward BSDE driven by a random measure, when the underlying forward process $X$ is a special semimartingale, or even more generally, a special weak Dirichlet process. Given a solution $(Y, Z, U)$, often $Y$ appears to be of the type $v\left(t, X_{t}\right)$ where $v$ is a deterministic function. In this paper, we identify $Z$ and $U$ in terms of $v$ applying stochastic calculus with respect to weak Dirichlet processes.

Indeed the employed techniques perform the calculus with respect to (special) weak Dirichlet processes developed in [5], extending the techniques established in the continuous framework in $[21,22]$. Given some filtration $\left(\mathcal{F}_{t}\right)$, we recall that a special weak Dirichlet process is a process of the type $X=M+A$, where $M$ is an $\left(\mathcal{F}_{t}\right)$-local martingale and $A$ is an $\left(\mathcal{F}_{t}\right)$-predictable orthogonal process, see Definition 5.6 in [5]. When $A$ has bounded variation, then $X$ is a special $\left(\mathcal{F}_{t}\right)$-semimartingale. That calculus has two important features: (1) the decomposition of a special weak Dirichlet process is unique, see Proposition 3.4; (2) there is a chain rule (in substitution of Itô's formula) allowing to expand $v\left(t, X_{t}\right)$, where $X$ is a special weak Dirichlet process of finite quadratic variation and $v$ is of class $C^{0,1}$, fulfilling some technical assumption, see Theorem 3.10. If we know a priori that $v\left(t, X_{t}\right)$ is the sum of a bounded variation process and a continuous $\left(\mathcal{F}_{t}\right)$-orthogonal process, then the chain rule does not require any differentiability on $v$; in that case, no assumptions are required on the càdlàg process $X$, see Proposition 3.15.

As we have already mentioned, we will focus on forward BSDEs, which constitute a particular case of BSDEs in their general form. BSDEs have been deeply studied since the seminal paper [31]. In [31], as well as in many subsequent papers, the standard Brownian motion is the driving process (Brownian context) and the concept of BSDE is based on a non-linear martingale 
representation theorem with respect to the corresponding Brownian filtration. A recent monograph on the subject is [33]. BSDEs driven by processes with jumps have also been investigated: two classes of such equations appear in the literature. The first one relates to BSDEs where the Brownian motion is replaced by a general càdlàg martingale $M$, see, among others, $[8,10,11,17]$. An alternative version of BSDEs with a discontinuous driving term is the one associated to an integer-valued random measure $\mu$, with corresponding compensator $v$. In this case, the BSDE is driven by a continuous martingale $M$ and a compensated random measure $\mu-v$. In that equation, naturally appears a purely discontinuous martingale which is a stochastic integral with respect to $\mu-v$, see, for example, $[1,3,4,9,39,40]$. A recent monograph on BSDEs driven by Poisson random measures is [16]. Connections between the martingale and the random measure driven BSDEs are illustrated by [28].

In this paper, we will focus on BSDEs driven by a compensated random measure $\mu-v$ (we will use the one-dimensional formalism for simplicity). We will not ask $\mu$ to be quasi-left-continuous, that is, $\mu(\{S\} \times \mathbb{R})=0$ on $\{S<\infty\}$, for every predictable time $S$, see the definition in Theorem (4.47) in [25]. Let $T>0$ be a finite horizon time. Besides $\mu$ and $v$ appear three driving random elements: a continuous martingale $M$, a non-decreasing adapted continuous process $\zeta$ and a predictable random measure $\lambda$ on $\Omega \times[0, T] \times \mathbb{R}$, equipped with the usual product $\sigma$-fields. Given a square integrable random variable $\xi$, and two measurable functions $\tilde{g}: \Omega \times[0, T] \times \mathbb{R}^{2} \rightarrow \mathbb{R}$, $\tilde{f}: \Omega \times[0, T] \times \mathbb{R}^{3} \rightarrow \mathbb{R}$, the equation takes the form

$$
\begin{aligned}
Y_{t}= & +\int_{] t, T]} \tilde{g}\left(s, Y_{s-}, Z_{s}\right) d \zeta_{s}+\int_{] t, T] \times \mathbb{R}} \tilde{f}\left(s, e, Y_{s-}, U_{s}(e)\right) \lambda(d s d e) \\
& -\int_{] t, T]} Z_{s} d M_{s}-\int_{] t, T] \times \mathbb{R}} U_{s}(e)(\mu-v)(d s d e) .
\end{aligned}
$$

As we have anticipated before, the unknown of (1.1) is a triplet $(Y, Z, U)$ where $Y, Z$ are adapted and $U$ is a predictable random field. The Brownian context of Pardoux-Peng appears as a particular case, setting $\mu=\lambda=0, \zeta_{s} \equiv s$. There, $M$ is a standard Brownian motion and $\xi$ is measurable with respect to the Brownian $\sigma$-field at terminal time. In that case the unknown can be reduced to $(Y, Z)$, since $U$ can be arbitrarily chosen. Another significant subcase of (1.1) arises when only the purely discontinuous driving term appears, that is, $M$ and $\zeta$ vanish.

The standard situation in the literature corresponds to the case when $\mu$ is quasi-left-continuous, see, for example, [6,39] when $\mu$ is a Poisson random measure, and [7] for a random measure $\mu$ more general than the Poisson one, whose compensator is absolutely continuous with respect to a deterministic measure. In the purely discontinuous subcase, for instance when $\mu$ is the jump measure of a marked point process, the well-posedness of the related BSDE can be settled by an iterative method, see [14]. Existence and uniqueness for BSDEs driven by a random measure $\mu$ which is not necessarily quasi-left-continuous are very recent, and have been discussed in [2] in the purely discontinuous case, and in a slightly different context by [12], for BSDEs driven by a countable sequence of square-integrable martingales.

When the random dependence of $\tilde{f}$ and $\tilde{g}$ is provided by a Markov solution $X$ of a forward SDE, and $\xi$ is a real function of $X$ at the terminal time $T$, then the BSDE (1.1) is called forward BSDE, as mentioned at the beginning. Forward BSDEs generally constitute stochastic representations of a partial integro-differential equation (PIDE). In the Brownian case, when $X$ is the 
solution of a classical SDE with diffusion coefficient $\sigma$, then the PIDE reduces to a semilinear parabolic PDE. If $v:[0, T] \times \mathbb{R} \times \mathbb{R}$ is a classical (smooth) solution of the mentioned PDE, then $Y_{s}=v\left(s, X_{s}\right), Z_{s}=\sigma\left(s, X_{s}\right) \partial_{x} v\left(s, X_{s}\right)$, generate a solution to the forward BSDE, see, for example, $[32,34,35]$. In the general case when the forward BSDEs are also driven by random measures, similar results have been established, for instance, by [6], for the jump-diffusion case, and by [13], for the purely discontinuous case, in particular when no Brownian noise appears. In the context of martingale driven forward BSDEs, a first approach to the probabilistic representation has been carried on in [30].

Conversely, solutions of forward BSDEs generate solutions of PIDEs in the viscosity sense. More precisely, for each given couple $(t, x) \in[0, T] \times \mathbb{R}$, consider an underlying process $X$ given by the solution $X^{t, x}$ of an SDE starting at $x$ at time $t$. Let $\left(Y^{t, x}, Z^{t, x}, U^{t, x}\right)$ be a family of solutions of the forward BSDE. In that case, under reasonable general assumptions, the function $v(t, x):=Y_{t}^{t, x}$ is a viscosity solution of the related PIDE. A demanding task consists in characterizing the couple $(Z, U):=\left(Z^{t, x}, U^{t, x}\right)$, in term of $v$; this is generally called the identification problem of $(Z, U)$. In the Brownian context, it was for instance the object of [20]: the authors show that if $v \in C^{0,1}$, then $Z_{s}=\sigma\left(s, X_{s}\right) \partial_{x} v\left(s, X_{s}\right)$; under more general assumptions, the authors also associate $Z$ with a generalized gradient of $v$. At our knowledge, in the general case, the problem of the identification of the martingale integrands couple $(Z, U)$ has not been deeply investigated, except for particular situations, as for instance the one treated in [14]: this problem was faced in [13].

A motivating PIDE is the one of Hamilton-Jacobi-Bellman related to stochastic optimal control problems, when the underlying is a general jump process. The solution to the identification problem in the related BSDE can be useful to determine feedback control strategies in verification theorems. Those verification theorems have the advantage of requiring less regularity of the value function than the classical ones, which need, instead, a time-space $C^{1,2}$ regularity. Another possible application concerns hedging in incomplete markets in mathematical finance. This can be treated making explicit the so called Föllmer-Schweizer decomposition of the payoff related to a contingent claim via mean-variance hedging; that decomposition is strictly related to a specific BSDE, as for instance illustrated in [28]. When the underlying is a general Markov process and the contingent claim is of vanilla type, solving the identification problem gives us suitable techniques to discuss mean-variance hedging.

In the present paper, we discuss the over-mentioned identification problem in a more general framework. In particular we have formulated a set of hypotheses which include the existing results in the literature. In Section 3, we state Theorems 3.8 and 3.14, which are the main results of the article. If $Y$ together with $(Z, U)$ constitutes a solution of a BSDE and there exists a function $v$ with some minimal regularity such that $Y_{t}=v\left(t, X_{t}\right)$, those two theorems allow to solve the identification problem. Their proof makes use in essential way of both features of the calculus related to weak Dirichlet processes. Indeed, first, supposing that $Y$ is a suitable $C^{0,1}$-deterministic function $v$ of the underlying process $X$, which is a special semimartingale $X$, related in a specific way to the random measure $\mu$, we apply the chain rule in Theorem 3.10. In particular, $Y$ will be a special weak Dirichlet process with prescribed local martingale part. Second, $Y$ can be decomposed using the fact that, together with $(Z, U)$, it solves the BSDE. So the uniqueness of decomposition of the special weak Dirichlet process $Y$ recalled in Proposition 3.4 allows us to identify the couple $(Z, U)$. This is the object of Theorem 3.8. On the other hand, in the purely discontinuous framework we make use instead of the chain rule Proposition 3.15. This does not 
even ask $X$ to be a special weak Dirichlet process, provided we have some a priori information on the structure of $v\left(t, X_{t}\right)$. At this point, by a similar argument, Theorem 3.14 also allows to tackle and solve the identification problem.

Section 4 is devoted to concrete applications in the following situations.

1. The case when the BSDE is driven by a Wiener process and a Poisson random measure and the underlying process $X$ is a jump-diffusion.

2. A case when the BSDE is driven by a quasi-left-continuous random measure and $X$ is a non-diffusive Markov processes. This case has also been treated with a different technique by [13].

3. A particular case of a BSDE driven by a non quasi-left-continuous random measure.

The paper is organized as follows. In Section 2.1, we fix the notations and we make some technical observations on stochastic integration with respect to a random measure; in Section 2.2 we introduce our basic set of hypotheses, and we provide some related technical results, which are proved in the Appendix. Section 3 is devoted to solve the identification problem. As mentioned earlier, applications are provided in Section 4.

\section{Notations and preliminaries}

In what follows, we are given a probability space $(\Omega, \mathcal{F}, \mathbb{P})$ a positive horizon $T$ and a filtration $\left(\mathcal{F}_{t}\right)_{t \geq 0}$, satisfying the usual conditions. Let $\mathcal{F}=\mathcal{F}_{T}$. Given a topological space $E$, in the sequel $\mathcal{B}(E)$ will denote the Borel $\sigma$-field associated with $E . \mathcal{P}$ (resp. $\tilde{\mathcal{P}}=\mathcal{P} \otimes \mathcal{B}(\mathbb{R})$ ) will denote the predictable $\sigma$-field on $\Omega \times[0, T]$ (resp. on $\tilde{\Omega}=\Omega \times[0, T] \times \mathbb{R}$ ). Analogously, we set $\mathcal{O}$ (resp. $\tilde{\mathcal{O}}=\mathcal{O} \otimes \mathcal{B}(\mathbb{R}))$ as the optional $\sigma$-field on $\Omega \times[0, T]($ resp. on $\tilde{\Omega})$. Moreover, $\tilde{\mathcal{F}}$ will be $\sigma$-field $\mathcal{F} \otimes \mathcal{B}([0, T] \times \mathbb{R})$, and we will indicate by $\mathcal{F}^{\mathbb{P}}$ the completion of $\mathcal{F}$ with the $\mathbb{P}$-null sets. We set $\tilde{\mathcal{F}}^{\mathbb{P}}=\mathcal{F}^{\mathbb{P}} \otimes \mathcal{B}([0, T] \times \mathbb{R})$. By default, all the stochastic processes will be considered with parameter $t \in[0, T]$. By convention, any càdlàg process defined on $[0, T]$ is extended to $\mathbb{R}_{+}$by continuity. A random set $A \subset \tilde{\Omega}$ is called evanescent if the set $\left\{\omega: \exists t \in \mathbb{R}_{+}\right.$with $\left.(\omega, t) \in A\right\}$ is $\mathbb{P}$-null. Generically, all the equalities of random sets will be intended up to an evanescent set.

A bounded variation process $X$ on $[0, T]$ will be said to be with integrable variation if the expectation of its total variation is finite. $\mathcal{A}$ (resp. $\mathcal{A}_{\text {loc }}$ ) will denote the collection of all adapted processes with integrable variation (resp. with locally integrable variation), and $\mathcal{A}^{+}$(resp. $\mathcal{A}_{\text {loc }}^{+}$) the collection of all adapted integrable increasing (resp. adapted locally integrable) processes. The significance of locally is the usual one which refers to localization by stopping times, see, for example, (0.39) of [25]. We will indicate by $C^{0,1}$ the space of all functions

$$
u:[0, T] \times \mathbb{R} \rightarrow \mathbb{R}, \quad(t, x) \mapsto u(t, x)
$$

that are continuous together their derivative $\partial_{x} u$.

\subsection{Stochastic integration with respect to integer-valued random measures}

The concept of random measure will be extensively used throughout the paper. For a detailed discussion on this topic and the unexplained notations, see Chapters I and II, Section 1, 
in [27], Chapter III in [25], and Chapter XI, Section 1, in [23]. In particular, if $\mu$ is a random measure on $[0, T] \times \mathbb{R}$, for any measurable real function $H$ defined on $\tilde{\Omega}$, one denotes $H \star \mu_{t}:=\int_{] 0, t] \times \mathbb{R}} H(\cdot, s, x) \mu(\cdot, d s d x)$, at least when the right-hand side is strictly greater than $-\infty$.

In the sequel of the section, $\mu$ will be an integer-valued random measure on $[0, T] \times \mathbb{R}$, and $v$ a "good" version of the compensator of $\mu$, as constructed in point (c) of Proposition 1.17, Chapter II, in [27]. Set $D=\{(\omega, t): \mu(\omega,\{t\} \times \mathbb{R})>0\}$, and

$$
J=\{(\omega, t): v(\omega,\{t\} \times \mathbb{R})>0\}, \quad K=\{(\omega, t): v(\omega,\{t\} \times \mathbb{R})=1\} .
$$

We define $v^{d}:=v \mathbb{1}_{J}$ and $v^{c}:=v \mathbb{1}_{J^{c}}$.

Remark 2.1. $J$ is the predictable support of $D$, see Proposition 1.14, Chapter II, in [27]. The definition of predictable support of a random set is given in Definition 2.32, Chapter I, in [27]. From that it follows that $\mathbb{1}_{J}={ }^{p}\left(\mathbb{1}_{D}\right) . K$ is the largest predictable subset of $D$, see Theorem 11.14 in [23]. Since $K$ is predictable, we have ${ }^{p}\left(\mathbb{1}_{K}\right)=\mathbb{1}_{K} . J$ is a thin set, see Proposition 2.34, Chapter I, in [27].

Remark 2.2. (i) $v$ admits a disintegration of the type

$$
\nu(\omega, d s d e)=d A_{s}(\omega) \phi(\omega, s, d e),
$$

where $\phi$ is a random kernel from $(\Omega \times[0, T], \mathcal{P})$ into $(\mathbb{R}, \mathcal{B}(\mathbb{R}))$ and $A$ is a right-continuous nondecreasing predictable process, such that $A_{0}=0$, see, for instance, Remark 4.4 in [2].

(ii) Given $v$ in the form (2.1), then the process $A$ is continuous if and only if, up to an evanescent set, $D=\bigcup_{n}\left[\left[T_{n}^{i}\right]\right],\left[\left[T_{n}^{i}\right]\right] \cap\left[\left[T_{m}^{i}\right]\right]=\varnothing, n \neq m$, where $\left(T_{n}^{i}\right)_{n}$ are totally inaccessible times, see, e.g., Assumption (A) in [14].

(iii) We recall that a totally inaccessible random time $T^{i}$ fulfills the property

$$
\mathbb{1}_{\left[\left[T^{i}\right]\right]}(\omega, S(\omega)) \mathbb{1}_{\{S<\infty\}}=0,
$$

for every predictable random time $S$, see Definition 2.20, Chapter I, in [27].

We recall an important notion of measure associated with $\mu$, given in formula (3.10) in [25].

Definition 2.3. Let $\left(\tilde{\Omega}_{n}\right)$ be a partition of $\tilde{\Omega}$ constituted by elements of $\tilde{\mathcal{O}}$, such that $\mathbb{1}_{\tilde{\Omega}_{n}} \star \mu \in \mathcal{A}$. $M_{\mu}^{\mathbb{P}}$ denotes the $\sigma$-finite measure on $\left(\tilde{\Omega}, \tilde{\mathcal{F}}^{\mathbb{P}}\right)$, such that for every $W: \tilde{\Omega} \rightarrow \mathbb{R}$ positive, bounded, $\tilde{\mathcal{F}}^{\mathbb{P}}$-measurable function,

$$
M_{\mu}^{\mathbb{P}}\left(W \mathbb{1}_{\tilde{\Omega}_{n}}\right)=\mathbb{E}\left[W \mathbb{1}_{\tilde{\Omega}_{n}} \star \mu_{T}\right]
$$

Let us now set $\hat{v}_{t}(d e):=v(\{t\}, d e)$ for all $t \in[0, T]$. For any $W \in \tilde{\mathcal{O}}$, we define

$$
\hat{W}_{t}=\int_{\mathbb{R}} W_{t}(x) \hat{v}_{t}(d e), \quad \tilde{W}_{t}=\int_{\mathbb{R}} W_{t}(x) \mu(\{t\}, d e)-\hat{W}_{t}, \quad t \geq 0,
$$


with the convention that $\tilde{W}_{t}=+\infty$ if $\hat{W}_{t}$ is not defined. For every $q \in[1, \infty$, we introduce the linear spaces

$$
\begin{aligned}
\mathcal{G}^{q}(\mu) & =\left\{W \in \tilde{\mathcal{P}}:\left[\sum_{s \leq .}\left|\tilde{W}_{s}\right|^{2}\right]^{q / 2} \in \mathcal{A}^{+}\right\}, \\
\mathcal{G}_{\mathrm{loc}}^{q}(\mu) & =\left\{W \in \tilde{\mathcal{P}}:\left[\sum_{s \leq .}\left|\tilde{W}_{s}\right|^{2}\right]^{q / 2} \in \mathcal{A}_{\mathrm{loc}}^{+}\right\} .
\end{aligned}
$$

Given $W \in \tilde{\mathcal{P}}$, we define the increasing (possibly infinite) predictable process

$$
C(W)_{t}=|W-\hat{W}|^{2} \star v_{t}+\sum_{s \leq t}\left(1-\hat{v}_{s}(\mathbb{R})\right)\left|\hat{W}_{s}\right|^{2},
$$

provided the right-hand side is well-defined. By Proposition 3.71 in [25], we have $\mathcal{G}^{2}(\mu)=\{W \in$ $\left.\tilde{\mathcal{P}}:\|W\|_{\mathcal{G}^{2}(\mu)}<\infty\right\}$, where

$$
\|W\|_{\mathcal{G}^{2}(\mu)}^{2}:=\mathbb{E}\left[C(W)_{T}\right]
$$

We also introduce the space

$$
\begin{aligned}
\mathcal{L}^{2}(\mu):= & \left\{W \in \tilde{\mathcal{P}}:\|W\|_{\mathcal{L}^{2}(\mu)}<\infty\right. \\
& \text { with } \left.\|W\|_{\mathcal{L}^{2}(\mu)}:=\mathbb{E}\left[\int_{] 0, T] \times \mathbb{R}}\left|W_{s}(e)\right|^{2} v(d s d e)\right]\right\} .
\end{aligned}
$$

Lemma 2.4. 1. If $W \in \mathcal{L}^{2}(\mu)$, then $W \in \mathcal{G}^{2}(\mu)$.

2. If $W \in \mathcal{L}_{\text {loc }}^{2}(\mu)$ then $W \in \mathcal{G}_{\text {loc }}^{2}(\mu)$.

Proof. Concerning item 1. it is enough to show that, if $W \in \mathcal{L}^{2}(\mu)$, then $\|W\|_{\mathcal{G}^{2}(\mu)}^{2} \leq\|W\|_{\mathcal{L}^{2}(\mu)}^{2}$. Let $W \in \tilde{\mathcal{P}}$. Recalling (2.3) and (2.4), we evaluate the expectation of the right-hand side in (2.3). For every $t \geq 0$, since $\hat{v}_{t}(\mathbb{R}) \leq 1$, we have

$$
\sum_{s \in] 0, t]}\left|\hat{W}_{s}\right|^{2}\left(1-\hat{v}_{s}(\mathbb{R})\right) \leq \sum_{s \leq t}\left|\hat{W}_{s}\right|^{2} \leq \sum_{s \leq t} \hat{v}_{s}(\mathbb{R}) \int_{\mathbb{R}}\left|W_{s}(e)\right|^{2} \hat{v}_{s}(d e) \leq|W|^{2} \star v_{t} .
$$

Moreover, taking into account that $|\hat{W}|^{2} \star v_{t}=\sum_{s \leq t}\left|\hat{W}_{s}\right|^{2} \hat{v}_{s}(\mathbb{R})$, for every $t \geq 0$, the process $C(W)$ defined in (2.3) can be decomposed as

$$
\begin{aligned}
C(W)_{t} & =|W|^{2} \star v_{t}-2 \sum_{s \leq t}\left|\hat{W}_{s}\right|^{2}+\sum_{s \leq t}\left|\hat{W}_{s}\right|^{2} \hat{v}_{s}(\mathbb{R})+\sum_{s \leq t}\left|\hat{W}_{s}\right|^{2}\left(1-\hat{v}_{s}(\mathbb{R})\right) \\
& =|W|^{2} \star v_{t}-\sum_{s \leq t}\left|\hat{W}_{s}\right|^{2} .
\end{aligned}
$$


In particular, since $W \in \mathcal{L}^{2}(\mu)$, we have

$$
\begin{aligned}
\|W\|_{\mathcal{G}^{2}(\mu)}^{2} & =\mathbb{E}\left[\int_{] 0, T] \times \mathbb{R}}\left|W_{s}(e)\right|^{2} v(d s d e)-\sum_{s \in] 0, T]}\left|\hat{W}_{s}\right|^{2}\right] \\
& \leq\|W\|_{\mathcal{L}^{2}(\mu)}^{2} .
\end{aligned}
$$

This concludes the proof of item 1. Item 2. follows by usual localization arguments.

Proposition 2.5. Let $\varphi: \Omega \times[0, T] \times \mathbb{R} \rightarrow \mathbb{R}$ be a $\tilde{\mathcal{P}}$-measurable function and $A$ a $\tilde{\mathcal{P}}$ measurable subset of $\Omega \times[0, T] \times \mathbb{R}$, such that

$$
\begin{aligned}
& |\varphi| \mathbb{1}_{A} \star \mu^{X} \in \mathcal{A}_{\mathrm{loc}}^{+}, \\
& |\varphi|^{2} \mathbb{1}_{A^{c} \star \mu^{X} \in \mathcal{A}_{\mathrm{loc}}^{+}} .
\end{aligned}
$$

Then the process $\varphi$ belongs to $\mathcal{G}_{\text {loc }}^{1}\left(\mu^{X}\right)$.

Proof. Formula (2.8), together with Proposition 1.28, Chapter II, in [27], gives that $\varphi \mathbb{1}_{A}$ belongs to $\mathcal{G}_{\text {loc }}^{1}\left(\mu^{X}\right)$. On the other hand, by Lemma 2.4-2., formula (2.9) implies that $\varphi \mathbb{1}_{A^{c}}$ belongs to $\mathcal{G}_{\mathrm{loc}}^{2}\left(\mu^{X}\right) \subset \mathcal{G}_{\mathrm{loc}}^{1}\left(\mu^{X}\right)$.

Remark 2.6. Since $\hat{W}=\hat{W} \mathbb{1}_{J}, \hat{v}(\mathbb{R}) \mathbb{1}_{K}=\mathbb{1}_{K}, 1-\hat{v}(\mathbb{R})>0$ on $J \backslash K$, the quantity $C(W)$ in (2.3) can be rewritten as

$$
C(W)=\left|W-\hat{W} \mathbb{1}_{J}\right|^{2} \star v+\sum_{s \leq \cdot}\left(1-\hat{v}_{S}(\mathbb{R})\right)\left|\hat{W}_{S}\right|^{2} \mathbb{1}_{J \backslash K}(s) .
$$

Proposition 2.7. If $D$ is the disjoint union of $K$ and $\bigcup_{n}\left[\left[T_{n}^{i}\right]\right]$, where $\left(T_{n}^{i}\right)_{n}$ are totally inaccessible finite times, then $J=K$ up to an evanescent set.

Proof. We start by noticing some basic facts. By Remark 2.1 , we have $\mathbb{1}_{J}={ }^{p}\left(\mathbb{1}_{D}\right)$, and ${ }^{p}\left(\mathbb{1}_{K}\right)=$ $\mathbb{1}_{K}$; on the other hand, by Theorem 5.2 in [23], for any totally inaccessible time $T^{i}$ we have that $\left.p_{\left(\left[T^{i}\right]\right]} \mathbb{1}_{\left\{T^{i}<\infty\right\}}\right)=0$, and therefore the predictable projection of $\mathbb{1}_{\left[\left[T_{n}^{i}\right]\right]}$ is zero since $T_{n}^{i}$ is a totally inaccessible finite time. Consequently, by additivity of the predictable projections, we

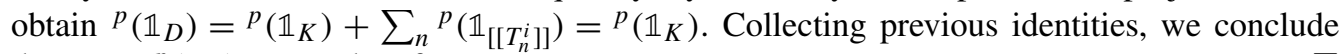
that $\mathbb{1}_{J}={ }^{p}\left(\mathbb{1}_{D}\right)=\mathbb{1}_{K}$, therefore $J=K$.

Proposition 2.8. If $C(W)_{T}=0$ a.s., then

$$
\left\|W-\hat{W} \mathbb{1}_{K}\right\|_{\mathcal{L}^{2}(\mu)}=0,
$$

or, equivalently, there exists a predictable process $\left(l_{s}\right)$ such that

$$
W_{s}(e)=l_{s} \mathbb{1}_{K}(s), \quad d \mathbb{P} v(d s d e) \text {-a.e. }
$$


In particular,

$$
W_{s}(e)=0, \quad d \mathbb{P} v^{c}(d s d e) \text {-a.e. },
$$

and there is a predictable process $\left(l_{s}\right)$ such that

$$
W_{s}(e)=l_{s} \mathbb{1}_{K}(s), \quad d \mathbb{P} v^{d}(d s d e) \text {-a.e. }
$$

Proof. By (2.10), we have

$$
\left\{\begin{array}{l}
\left|W-\hat{W} \mathbb{1}_{J}\right|^{2} \star v=0, \\
\sum_{s \leq \cdot}\left(1-\hat{v}_{s}(\mathbb{R})\right)\left|\hat{W}_{s}\right|^{2} \mathbb{1}_{J \backslash K}(s)=0 .
\end{array}\right.
$$

Since $1-\hat{v}(E)>0$ on $J \backslash K$, previous identities give

$$
\left\{\begin{array}{l}
\left|W-\hat{W} \mathbb{1}_{J}\right|^{2} \star v=0, \\
\hat{W} \mathbb{1}_{J \backslash K}=0,
\end{array}\right.
$$

and this gives (2.11). We show now the equivalence property. Obviously (2.11) implies (2.12)

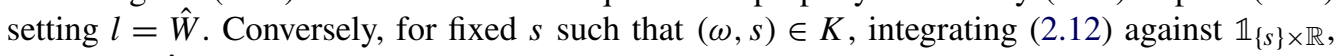
we obtain $\hat{W}_{s} \mathbb{1}_{K}(s)=l_{s} \mathbb{1}_{K}(s)$, which implies (2.11). Finally, (2.13) and (2.14) follow observing that $\left|W-l \mathbb{1}_{K}\right|^{2} \star v=|W|^{2} \star v^{c}+\left|W-l \mathbb{1}_{K}\right|^{2} \star v^{d}$.

\subsection{A class of stochastic processes $X$ related in a specific way to an integer-valued random measure $\mu$}

We will formulate two assumptions related to an integer-valued random measure $\mu$ on $[0, T] \times \mathbb{R}$ and some càdlàg process $X$. We recall that a sequence of random times $\left(T_{n}\right)_{n}$ exhausts the jumps of a process $Y$ if $\left[\left[T_{n}\right]\right] \cap\left[\left[T_{m}\right]\right]=\varnothing, n \neq m$, and $\{\Delta Y \neq 0\}=\bigcup_{n}\left[\left[T_{n}\right]\right]$, see Definition 1.30, Chapter I, in [27]. A process $Y$ is quasi-left-continuous if and only if there is a sequence of totally inaccessible times $\left(T_{n}^{i}\right)$ that exhausts the jumps of $Y$, see Proposition 2.26, Chapter I, in [27].

Hypothesis 2.9. $X$ is an adapted càdlàg process with decomposition $X=X^{i}+X^{p}$, where

1. $Y:=X^{i}$ is a càdlàg quasi-left-continuous adapted process satisfying $\{\Delta Y \neq 0\} \subset D$. Moreover, there exists a $\tilde{\mathcal{P}}$-measurable map $\tilde{\gamma}: \Omega \times] 0, T] \times \mathbb{R} \rightarrow \mathbb{R}$ such that

$$
\Delta Y_{t}(\omega) \mathbb{1}_{] 0, T]}(t)=\tilde{\gamma}(\omega, t, \cdot) \quad d M_{\mu}^{\mathbb{P}} \text {-a.e. }
$$

2. $X^{p}$ is a càdlàg predictable process satisfying $\left\{\Delta X^{p} \neq 0\right\} \subset J$.

Remark 2.10. Theorem 3.89 in [25] states an Itô formula which transforms a special semimartingale $X$ into a special semimartingale $F\left(X_{t}\right)$ through a $C^{2}$ function $F: \mathbb{R} \rightarrow \mathbb{R}$. There, the process $Y=X$ is supposed to fulfill Hypothesis 2.9-1. 
Remark 2.11. If $\mu$ is the jump measure of a càdlàg process $X$, then Hypothesis 2.9-1. holds for $Y=X$, with $\tilde{\gamma}(t, \omega, x)=x$.

The proof of the following result is reported in Section B.1.

Proposition 2.12. Let $X$ be a process verifying Hypothesis 2.9. Then, there exists a null set $\mathcal{N}$ such that, for every Borel function $\varphi:[0, T] \times \mathbb{R} \rightarrow \mathbb{R}_{+}$satisfying $\varphi(s, 0)=0, s \in[0, T]$, we have, for every $\omega \notin \mathcal{N}$,

$$
\int_{] 0, T] \times \mathbb{R}} \varphi(s, x) \mu^{X}(\omega, d s d x)=\int_{] 0, T] \times \mathbb{R}} \varphi(s, \tilde{\gamma}(\omega, s, e)) \mu(\omega, d s d e)+V^{\varphi}(\omega),
$$

with $V^{\varphi}(\omega)=\sum_{0<s \leq T} \varphi\left(s, \Delta X_{s}^{p}(\omega)\right)$. In particular,

$$
\begin{aligned}
& \int_{] 0, T] \times \mathbb{R}} \varphi(s, x) \mu^{X}(\omega, d s d x) \\
& \quad \geq \int_{] 0, T] \times \mathbb{R}} \varphi(s, \tilde{\gamma}(\omega, s, e)) \mu(\omega, d s d e) \quad \text { for every } \omega \notin \mathcal{N} .
\end{aligned}
$$

Identity (2.16) still holds true when $\varphi:[0, T] \times \mathbb{R} \rightarrow \mathbb{R}$ and the left-hand side is finite

Remark 2.13. The result in Proposition 2.12 still holds true if $\varphi$ is a real-valued random function on $\Omega \times[0, T] \times \mathbb{R}$.

We will make use in the sequel of the following assumption on $\mu$.

Hypothesis 2.14. (i) $D=K \cup\left(\bigcup_{n}\left[\left[T_{n}^{i}\right]\right]\right)$ up to an evanescent set, where $\left(T_{n}^{i}\right)_{n}$ are totally inaccessible times such that $\left[\left[T_{n}^{i}\right]\right] \cap\left[\left[T_{m}^{i}\right]\right]=\varnothing, n \neq m$;

(ii) for every predictable time $S$ such that $[[S]] \subset K, v(\{S\}$, de $)=\mu(\{S\}$, de) a.s.

Remark 2.15. Hypothesis 2.14(i) implies that $J=K$, up to an evanescent set, see Proposition 2.7 .

Remark 2.16. 1. If $\hat{v}=0$, then $J=K=\varnothing$. Taking into account Remark 2.2(ii), $D=\bigcup_{n}\left[\left[T_{n}^{i}\right]\right]$, $\left[\left[T_{n}^{i}\right]\right] \cap\left[\left[T_{m}^{i}\right]\right]=\varnothing, n \neq m$, where $\left(T_{n}^{i}\right)_{n}$ are totally inaccessible times, and Hypothesis 2.14 trivially holds.

2. Notice that, if $v$ is given in the form (2.1) and the process $A$ appearing in (2.1) is continuous, then $\hat{v}=0$. In that case, Theorem (4.47) of [25] states that $\mu$ is quasi-left-continuous.

We have the following important technical result, for the proof see Section B.2.

Proposition 2.17. Let $\mu$ satisfy Hypothesis 2.14. Assume that $X$ is a process verifying Hypothesis 2.9. Let $\varphi: \Omega \times[0, T] \times \mathbb{R} \rightarrow \mathbb{R}_{+}$such that $\varphi(\omega, s, 0)=0$ for every $s \in[0, T]$, up to indistin- 
guishability, and assume that there exists a $\tilde{\mathcal{P}}$-measurable subset $A$ of $\Omega \times[0, T] \times \mathbb{R}$ satisfying

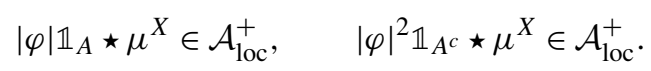

Then

$$
\int_{[0, \cdot] \times \mathbb{R}} \varphi(s, x)\left(\mu^{X}-v^{X}\right)(d s d x)=\int_{] 0, \cdot] \times \mathbb{R}} \varphi(s, \tilde{\gamma}(s, e))(\mu-v)(d s d e) .
$$

Remark 2.18. Under condition (2.18), Proposition 2.5 and inequality (2.17) in Proposition 2.12 imply that $(s, x) \mapsto \varphi(s, x) \in \mathcal{G}_{\text {loc }}^{1}\left(\mu^{X}\right)$ and $(s, e) \mapsto \varphi(s, \tilde{\gamma}(s, e)) \in \mathcal{G}_{\text {loc }}^{1}(\mu)$. In particular the two stochastic integrals in (2.19) are well-defined.

We end the section focusing on the case when $X$ is of jump-diffusion type. The following result is proved in Section B.3.

Lemma 2.19. Let $\mu$ satisfy Hypothesis 2.14. Let $N$ be a continuous martingale, and $B$ an increasing predictable càdlàg process, with $B_{0}=0$, such that $\{\Delta B \neq 0\} \subset J$. Let $X$ be a process which is solution of equation

$$
\begin{aligned}
X_{t}= & X_{0}+\int_{0}^{t} b\left(s, X_{s-}\right) d B_{s}+\int_{0}^{t} \sigma\left(s, X_{s}\right) d N_{s} \\
& +\int_{] 0, t] \times \mathbb{R}} \gamma\left(s, X_{s-}, e\right)(\mu-v)(d s d e),
\end{aligned}
$$

for some given Borel functions $b, \sigma:[0, T] \times \mathbb{R} \rightarrow \mathbb{R}$, and $\gamma:[0, T] \times \mathbb{R} \times \mathbb{R} \rightarrow \mathbb{R}$ such that

$$
\begin{aligned}
\int_{0}^{t}\left|b\left(s, X_{s-}\right)\right| d B_{s} & <\infty \quad \text { a.s., } \\
\int_{0}^{t}\left|\sigma\left(s, X_{s}\right)\right|^{2} d[N, N]_{s} & <\infty \quad \text { a.s., } \\
(\omega, s, e) & \mapsto \gamma\left(s, X_{s-}(\omega), e\right) \in \mathcal{G}_{\text {loc }}^{1}(\mu) .
\end{aligned}
$$

Then $X$ satisfies Hypothesis 2.9 with decomposition $X=X^{i}+X^{p}$, where

$$
\begin{aligned}
X_{t}^{i} & =\int_{] 0, t] \times \mathbb{R}} \gamma\left(s, X_{s-}, e\right)(\mu-v)(d s d e), \\
X_{t}^{p} & =X_{0}+\int_{0}^{t} b\left(s, X_{s-}\right) d B_{s}+\int_{0}^{t} \sigma\left(s, X_{s}\right) d N_{s}, \\
\tilde{\gamma}(\omega, s, e) & =\gamma\left(s, X_{s-}(\omega), e\right)\left(1-\mathbb{1}_{K}(\omega, s)\right) .
\end{aligned}
$$




\section{BSDEs driven by an integer-valued random measure}

Let $\mu$ be an integer-valued random measure defined on $[0, T] \times \mathbb{R}$. Let $M$ be a continuous process with $M_{0}=0$. Let $\left(\mathcal{F}_{t}\right)$ be the canonical filtration associated to $\mu$ and $M$, and suppose that $M$ is an $\left(\mathcal{F}_{t}\right)$-local martingale. $v$ will denote a "good" version of the dual predictable projection of $\mu$ in the sense of Proposition 1.17, Chapter II, in [27]. In particular, $v(\omega,\{t\} \times \mathbb{R}) \leq 1$ identically. Let $\lambda$ be a predictable random measure on $[0, T] \times \mathbb{R}$, and $\zeta$ a non-decreasing adapted continuous process.

We focus now on the general BSDE (1.1) of the Introduction, whose coefficients are the following: $\xi$ is an $\mathcal{F}_{T}$-measurable square integrable random variable, $\tilde{f}: \Omega \times[0, T] \times \mathbb{R}^{3} \rightarrow \mathbb{R}$ (resp. $\tilde{g}: \Omega \times[0, T] \times \mathbb{R}^{2} \rightarrow \mathbb{R}$ ) is a measurable function, whose domain is equipped with the $\sigma$-field $\mathcal{F} \otimes \mathcal{B}\left([0, T] \times \mathbb{R}^{3}\right)\left(\right.$ resp. $\mathcal{F} \otimes \mathcal{B}\left([0, T] \times \mathbb{R}^{2}\right)$ ).

A solution of BSDE (1.1) is a triple of processes $(Y, Z, U)$ such that the first two integrals in (1.1) exist and are finite in the Lebesgue sense, $Y$ is adapted and càdlàg, $Z$ is progressively measurable with $Z \in L^{2}\left([0, T], d\langle M\rangle_{t}\right)$ a.s., and $U \in \mathcal{G}_{\text {loc }}^{2}(\mu)$.

Remark 3.1. Uniqueness in $\mathcal{G}_{\text {loc }}^{2}(\mu)$ means the following: if $(Y, Z, U),\left(Y^{\prime}, Z^{\prime}, U^{\prime}\right)$ are solutions of the BSDE (1.1), then $Y=Y^{\prime} \lambda(d s, \mathbb{R})$ and $d \zeta_{s}$-a.e., for almost all $\omega, Z=Z^{\prime} d \mathbb{P} d\langle M\rangle_{t}$ a.e., and there is a predictable process $\left(l_{t}\right)$ such that $U_{t}(e)-U_{t}^{\prime}(e)=l_{t} \mathbb{1}_{K}(t), d \mathbb{P} v(d t d e)$-a.e. The latter fact is a direct consequence of Proposition 2.8. Moreover, provided that $\lambda \ll v$, given a solution $\left(Y, Z, U_{0}\right)$ of $\operatorname{BSDE}(1.1)$, the class of all solutions will be given by the triples $(Y, Z, U)$, where $U=l \mathbb{1}_{K}+U_{0}$ for some predictable process $\left(l_{t}\right)$. In particular, if $K=\varnothing$, then the third component of the BSDE solution is uniquely characterized in $\mathcal{L}^{2}(\mu)$.

Remark 3.2. A general BSDE of type (1.1) is considered for instance, in [40], with the following restrictions on the random measures $\lambda$ and $\nu$ :

$$
\begin{aligned}
& \lambda([0, T] \times \mathbb{R}) \text { is a bounded random variable, } \lambda([0, t] \times \mathbb{R}) \text { is continuous w.r.t. } t \\
& v([0, t] \times \mathbb{R}) \text { is continuous w.r.t. } t \text {. }
\end{aligned}
$$

In Theorem 3.2 in [40], the author proves that under suitable assumptions on the coefficients $(\xi, \tilde{f}, \tilde{g})$ there exists a unique triplet of processes $(Y, Z, U) \in \mathcal{L}^{2}(\zeta \lambda) \times \mathcal{L}^{2}(M) \times \mathcal{L}^{2}(\mu)$, with $\mathbb{E}\left[\sup _{t \in[0, T]} Y_{t}^{2}\right]<\infty$, satisfying BSDE (1.1), where

$$
\begin{aligned}
& \mathcal{L}^{2}(\zeta \lambda):=\left\{\left(Y_{t}\right)_{t \in[0, T]} \text { optional: } \mathbb{E}\left[\int_{0}^{T} Y_{s}^{2} d \zeta_{s}\right]+\mathbb{E}\left[\int_{0}^{T} Y_{s}^{2} \lambda(d s, \mathbb{R})\right]<\infty\right\}, \\
& \mathcal{L}^{2}(M):=\left\{\left(Z_{t}\right)_{t \in[0, T]} \text { predictable: } \mathbb{E}\left[\int_{0}^{T} Z_{s}^{2} d\langle M\rangle_{s}\right]<\infty\right\},
\end{aligned}
$$

and $\mathcal{L}^{2}(\mu)$ is the space introduced in (2.5). 
When $\zeta$ and $M$ vanish, BSDE (1.1) turns out to be driven only by a purely discontinuous martingale, and becomes

$$
Y_{t}=\xi+\int_{] t, T] \times \mathbb{R}} \tilde{f}\left(s, e, Y_{s-}, U_{s}(e)\right) \lambda(d s d e)-\int_{] t, T] \times \mathbb{R}} U_{s}(e)(\mu-v)(d s d e) .
$$

Remark 3.3. The process $Y$ solution to (3.2) is an $\left(\mathcal{F}_{t}\right)$-orthogonal process. In fact, for every continuous $\left(\mathcal{F}_{t}\right)$-local martingale $N$ we have

$$
\begin{aligned}
{[Y, N]_{t}=} & {\left[\int_{] 0, \cdot] \times \mathbb{R}} \tilde{f}\left(s, e, Y_{s-}, U_{s}(e)\right) \lambda(d s, d e), N\right]_{t} } \\
& -\left[\int_{] 0, \cdot] \times \mathbb{R}} U_{s}(e)(\mu-v)(d s d e), N\right]_{t}
\end{aligned}
$$

Since $\int_{] 0, \cdot] \times \mathbb{R}} \tilde{f}\left(s, e, Y_{s-}, U_{s}(e)\right) \lambda(d s, d e)$ is a bounded variation process, the first bracket in the right-hand side of (3.3) is zero by Proposition 2.14 in [5]. On the other hand, the second term in the right-hand side of (3.3) is zero because $\int_{] 0, \cdot] \times \mathbb{R}} U_{s}(e)(\mu-v)(d s d e)$ is a purely discontinuous martingale.

\subsection{Identification of the BSDEs solution}

The fundamental tool of this section is the notion of covariation of two processes $X$ and $Y$ (that are not necessarily semimartingales), denoted [X,Y], see Definition 2.4 in [5]. A process $X$ is said to be finite quadratic variation process if $[X, X]$ exists. Any $\left(\mathcal{F}_{t}\right)$-adapted process $X$ is said to be $\left(\mathcal{F}_{t}\right)$-orthogonal if $[X, N]=0$ for every $N$ continuous local $\left(\mathcal{F}_{t}\right)$-martingale.

By Proposition 5.9 in [5], we have the following result.

Proposition 3.4. Any $\left(\mathcal{F}_{t}\right)$-special weak Dirichlet process $X$ admits a decomposition of the type

$$
X=X^{c}+M^{d}+A,
$$

where $X^{c}$ is a continuous local martingale, $M^{d}$ is a purely discontinuous local martingale, and $A$ is an $\left(\mathcal{F}_{t}\right)$-predictable and orthogonal process, with $A_{0}=0$. Equation (3.4) is called the canonical decomposition of $X$.

The following condition on $X$ will play a fundamental role in the sequel:

$$
\int_{] 0, \cdot] \times \mathbb{R}}|x| \mathbb{1}_{\{|x|>1\}} \mu^{X}(d s d x) \in \mathcal{A}_{\mathrm{loc}}^{+} .
$$

Moreover, we will be interested in functions $v:[0, T] \times \mathbb{R} \rightarrow \mathbb{R}$ fulfilling the integrability property

$$
\int_{] 0, \cdot] \times \mathbb{R}}\left|v\left(s, X_{s-}+x\right)-v\left(s, X_{s-}\right)-x \partial_{x} v\left(s, X_{s-}\right)\right| \mathbb{1}_{\{|x|>1\}} \mu^{X}(d s d x) \in \mathcal{A}_{\mathrm{loc}}^{+} .
$$


Remark 3.5. By Proposition 4.5 in [5], if $X$ is a càdlàg process such that $\sum_{s \leq T}\left|\Delta X_{S}\right|^{2}<\infty$ a.s., and $v:[0, T] \times \mathbb{R} \rightarrow \mathbb{R}$ is a function of class $C^{0,1}$, then

$$
\left|v\left(s, X_{s-}+x\right)-v\left(s, X_{s-}\right)\right|^{2} \mathbb{1}_{\{|x| \leq 1\}} \star \mu^{X} \in \mathcal{A}_{\mathrm{loc}}^{+} .
$$

Moreover, Lemma 5.29 in [5] states that if $X$ is a càdlàg process satisfying condition (3.5), and $v:[0, T] \times \mathbb{R} \rightarrow \mathbb{R}$ is a function of class $C^{0,1}$ fulfilling (3.6), then

$$
\left|v\left(s, X_{s-}+x\right)-v\left(s, X_{s-}\right)\right| \mathbb{1}_{\{|x|>1\}} \star \mu^{X} \in \mathcal{A}_{\mathrm{loc}}^{+} .
$$

We have the following result.

Proposition 3.6. Let $X$ be a process such that $(X, \mu)$ verifies Hypothesis 2.9. Let in addition $v:[0, T] \times \mathbb{R} \rightarrow \mathbb{R}$ be a function of class $C^{0,1}$. If $X$ and $v$ satisfy conditions (3.5) and (3.6), and moreover $\sum_{s \leq T}\left|\Delta X_{s}\right|^{2}<\infty$ a.s., then

$$
(s, e) \mapsto v\left(s, X_{s-}+\tilde{\gamma}(s, e)\right)-v\left(s, X_{s-}\right) \in \mathcal{G}_{\text {loc }}^{1}(\mu) .
$$

Proof. Step 1. We first notice that, if $\sum_{s \leq T}\left|\Delta X_{s}\right|^{2}<\infty$ a.s., then

$$
\left|v\left(s, X_{s-}+\tilde{\gamma}(s, e)\right)-v\left(s, X_{s-}\right)\right|^{2} \mathbb{1}_{\{|\tilde{\gamma}(s, e)| \leq 1\}} \star \mu \in \mathcal{A}_{\mathrm{loc}}^{+} .
$$

Indeed, this follows from (3.7) and inequality (2.17) in Proposition 2.12, with $\varphi(\omega, s, x)=$ $\left|v\left(s, X_{s-}(\omega)+x\right)-v\left(s, X_{s-}(\omega)\right)\right|^{2} \mathbb{1}_{\{|x| \leq 1\}}$, taking into account Remark 2.13.

Step 2. We observe that, if $X$ and $v$ satisfy conditions (3.5) and (3.6), then

$$
\left|v\left(s, X_{s-}+\tilde{\gamma}(s, e)\right)-v\left(s, X_{s-}\right)\right| \mathbb{1}_{\{|\tilde{\gamma}(s, e)|>1\}} \star \mu \in \mathcal{A}_{\text {loc }}^{+} .
$$

This is a consequence (3.8), (3.5) and (3.6) together with inequality (2.17) in Proposition 2.12, with $\varphi(\omega, s, x)=\left|v\left(s, X_{s-}(\omega)+x\right)-v\left(s, X_{s-}(\omega)\right)\right| \mathbb{1}_{\{|x|>1\}}$, taking into account Remark 2.13. Step 3. The conclusion of Proposition 3.6 is a direct consequence of Steps 1 and 2, together with Proposition 2.5, with $\varphi(\omega, s, e)=v\left(s, X_{s-}(\omega)+\tilde{\gamma}(\omega, s, e)\right)-v\left(s, X_{s-}(\omega)\right)$ and $A=\{(\omega, s, e)$ : $|\tilde{\gamma}(\omega, s, e)|>1\}$.

Let us now consider the following assumption on a couple $(X, Y)$ of adapted processes.

Hypothesis 3.7. $X$ is a special weak Dirichlet process of finite quadratic variation, satisfying condition (3.5). $Y_{t}=v\left(t, X_{t}\right)$ for some (deterministic) function $v:[0, T] \times \mathbb{R} \rightarrow \mathbb{R}$ of class $C^{0,1}$ such that $v$ and $X$ verify condition (3.6).

Our first main result is the following.

Theorem 3.8. Let $\mu$ satisfy Hypothesis 2.14 , and assume that $X$ is a process such that $(X, \mu)$ verifies Hypothesis 2.9. Let $(Y, Z, U)$ be a solution to the BSDE (1.1) such that the pair $(X, Y)$ 
satisfies Hypothesis 3.7 with corresponding function $v$. Let $X^{c}$ denote the continuous local martingale of $X$ given in the canonical decomposition (3.4).

Then, the pair $(Z, U)$ fulfills

$$
\begin{aligned}
Z_{t} & =\partial_{x} v\left(t, X_{t}\right) \frac{d\left\langle X^{c}, M\right\rangle_{t}}{d\langle M\rangle_{t}} \quad d \mathbb{P} d\langle M\rangle_{t} \text {-a.e., } \\
\int_{] 0, t] \times \mathbb{R}} H_{s}(e)(\mu-v)(d s d e) & =0, \quad \forall t \in] 0, T], \text { a.s., }
\end{aligned}
$$

with

$$
H_{s}(e):=U_{s}(e)-\left(v\left(s, X_{s-}+\tilde{\gamma}(s, e)\right)-v\left(s, X_{s-}\right)\right) .
$$

If, in addition, $H \in \mathcal{G}_{\mathrm{loc}}^{2}(\mu)$, then there exists a predictable process $\left(l_{s}\right)$ such that

$$
H_{S}(e)=l_{s} \mathbb{1}_{K}(s), \quad d \mathbb{P} v(d s d e) \text {-a.e. }
$$

In particular,

$$
H_{s}(e)=0, \quad d \mathbb{P} v^{c}(d s d e) \text {-a.e. }
$$

and

$$
H_{s}(e)=l_{s} \mathbb{1}_{K}(s), \quad d \mathbb{P} v^{d}(d s d e) \text {-a.e. }
$$

Remark 3.9. Notice that $H$ in (3.11) belongs to $\mathcal{G}_{\text {loc }}^{1}(\mu)$, so that the integral in (3.10) is welldefined. Indeed, by Hypothesis 3.7, $X$ and $v$ in the statement of Theorem 3.8 satisfy (3.5) and (3.6). By Proposition 3.6, it follows that $(s, e) \mapsto\left(v\left(s, X_{s-}+\tilde{\gamma}(s, e)\right)-v\left(s, X_{s-}\right)\right) \in \mathcal{G}_{\text {loc }}^{1}(\mu)$. Since $U \in \mathcal{G}_{\text {loc }}^{2}(\mu) \subset \mathcal{G}_{\text {loc }}^{1}(\mu)$, the conclusion follows.

The proof of Theorem 3.8 is based essentially on the following stability result for càdlàg processes, which was the object of Theorem 5.31 in [5].

Theorem 3.10. Let $X$ be an $\left(\mathcal{F}_{t}\right)$-special weak Dirichlet process of finite quadratic variation with its canonical decomposition $X=X^{c}+M^{d}+A$, satisfying condition (3.5). Then, for every $v:[0, T] \times \mathbb{R} \rightarrow \mathbb{R}$ of class $C^{0,1}$ verifying (3.6), we have

$$
\begin{aligned}
v\left(t, X_{t}\right)= & v\left(0, X_{0}\right)+\int_{0}^{t} \partial_{x} v\left(s, X_{s}\right) d X_{s}^{c} \\
& +\int_{] 0, t] \times \mathbb{R}}\left(v\left(s, X_{s-}+x\right)-v\left(s, X_{s-}\right)\right)\left(\mu^{X}-v^{X}\right)(d s d x)+A^{v}(t),
\end{aligned}
$$

where $A^{v}$ is a predictable $\left(\mathcal{F}_{t}\right)$-orthogonal process.

Proof of Theorem 3.8. By assumption, $X$ is a special weak Dirichlet process satisfying condition (3.5), and $v$ is a function of class $C^{0,1}$ satisfying the integrability condition (3.6). So we are 
in the condition to apply Theorem 3.10 to $v\left(t, X_{t}\right)$. We set $\varphi(s, x):=v\left(s, X_{s-}+x\right)-v\left(s, X_{s-}\right)$. Since $X$ is of finite quadratic variation and verifies (3.5), and $X$ and $v$ satisfy (3.6), by (3.7) and (3.8) we see that the process $\varphi$ verifies condition (2.18) with $A=\{|x|>1\}$. Moreover, $\varphi(s, 0)=0$. Since $\mu$ verifies Hypothesis 2.14 and $X$ verifies Hypothesis 2.9 , we can apply Proposition 2.17 to $\varphi(s, x)$. Identity (3.14) becomes

$$
\begin{aligned}
v\left(t, X_{t}\right)= & v\left(0, X_{0}\right)+\int_{] 0, t] \times \mathbb{R}}\left(v\left(s, X_{s-}+\tilde{\gamma}(s, e)\right)-v\left(s, X_{s-}\right)\right)(\mu-v)(d s d e) \\
& +\int_{] 0, t]} \partial_{x} v\left(s, X_{s}\right) d X_{s}^{c}+A^{v}(t) .
\end{aligned}
$$

At this point we recall that the process $Y_{t}=v\left(t, X_{t}\right)$ fulfills the BSDE (1.1), which can be rewritten as

$$
\begin{aligned}
Y_{t}= & Y_{0}+\int_{] 0, t]} Z_{s} d M_{s}+\int_{] 0, t] \times \mathbb{R}} U_{s}(e)(\mu-v)(d s d e) \\
& -\int_{] 0, t]} \tilde{g}\left(s, Y_{s-}, Z_{s}\right) d \zeta_{s}-\int_{] 0, t] \times \mathbb{R}} \tilde{f}\left(s, e, Y_{s-}, U_{s}(e)\right) \lambda(d s d e) .
\end{aligned}
$$

By Proposition 3.4 the uniqueness of the decomposition (3.15) yields identity (3.10) and

$$
\int_{] 0, t]} Z_{s} d M_{s}=\int_{] 0, t]} \partial_{x} v\left(s, X_{s}\right) d X_{s}^{c} .
$$

In particular, from (3.17) we get

$$
\begin{aligned}
0 & =\left\langle\int_{] 0, t]} Z_{s} d M_{s}-\int_{] 0, t]} \partial_{x} v\left(s, X_{s}\right) d X_{s}^{c}, M_{t}\right\rangle \\
& =\int_{] 0, t]} Z_{s} d\langle M\rangle_{s}-\int_{] 0, t]} \partial_{x} v\left(s, X_{s}\right) \frac{d\left\langle X^{c}, M\right\rangle_{s}}{d\langle M\rangle_{s}} d\langle M\rangle_{s} \\
& =\int_{] 0, t]}\left(Z_{s}-\partial_{x} v\left(s, X_{s}\right) \frac{d\left\langle X^{c}, M\right\rangle_{s}}{d\langle M\rangle_{s}}\right) d\langle M\rangle_{s},
\end{aligned}
$$

that gives identification (3.9).

If in addition we assume that $H \in \mathcal{G}_{\text {loc }}^{2}(\mu)$, the predictable bracket at time $t$ of the purely discontinuous martingale in identity (3.10) is well-defined, and equals $C(H)$ by Theorem 11.21, point (3), in [23]. Since $C(H)_{T}=0$ a.s., the conclusion follows from Proposition 2.8.

Let us now consider a BSDE driven only by a purely discontinuous martingale, of the form (3.2). We formulate the following assumption for a couple of adapted processes $(X, Y)$.

We first introduce some notations. Let $E$ be a closed subset of $\mathbb{R}$ on which $X$ takes values. Given a càdlàg function $\varphi:[0, T] \rightarrow \mathbb{R}$, we denote by $\mathcal{C}_{\varphi}$ the set of times $t \in[0, T]$ for which there is a left (resp. right) neighborhood $\left.I_{t-}=\right] t-\varepsilon, t$ [ (resp. $I_{t+}=[t, t+\varepsilon[$ ) such that $\varphi$ is constant on $I_{t-}$ and $I_{t+}$. 
Hypothesis 3.11. (i) $Y$ is an $\left(\mathcal{F}_{t}\right)$-orthogonal process such that $\sum_{s \leq T}\left|\Delta Y_{s}\right|<\infty$, a.s.

(ii) $X$ is a càdlàg process and $Y_{t}=v\left(t, X_{t}\right)$ for some deterministic function $v:[0, T] \times \mathbb{R} \rightarrow$ $\mathbb{R}$, satisfying the integrability condition

$$
\int_{] 0, \cdot] \times \mathbb{R}}\left|v\left(t, X_{t-}+x\right)-v\left(t, X_{t-}\right)\right| \mu^{X}(d t d x) \in \mathcal{A}_{\mathrm{loc}}^{+} .
$$

(iii) There exists $\mathcal{C} \in[0, T]$ such that for $\omega$ a.s. $\mathcal{C} \supset \mathcal{C}_{X}(\omega)$, and

- $\forall t \in \mathcal{C}, t \mapsto v(t, x)$ is continuous $\forall x \in E$;

- $\forall t \in \mathcal{C}^{c}, x \in E,(t, x)$ is a continuity point of $v$.

Remark 3.12. Item (iii) of Hypothesis 3.11 is fulfilled in two typical situations.

1. $\mathcal{C}=[0, T]$. Almost surely $X$ admits a finite number of jumps and $t \mapsto v(t, x)$ is continuous $\forall x \in E$.

2. $\mathcal{C}=\varnothing$ and $\left.v\right|_{[0, T] \times E}$ is continuous.

Remark 3.13. Assume that Hypothesis 3.11(iii) holds. Then

(i) $Y_{t}=v\left(t, X_{t}\right)$ is necessarily a càdlàg process.

(ii) $\forall t \in[0, T], \Delta Y_{t}=v\left(t, X_{t}\right)-v\left(t, X_{t-}\right)$.

Our second main result is the following.

Theorem 3.14. Let $\mu$ satisfy Hypothesis 2.14 , and assume that $X$ is a process such that $(X, \mu)$ verifies Hypothesis 2.9. Let $(Y, U)$ be a solution to the BSDE (3.2), such that $(X, Y)$ satisfies Hypothesis 3.11 with corresponding function $v$. Then, the random field $U$ satisfies

$$
\left.\left.\int_{] 0, t] \times \mathbb{R}} H_{S}(e)(\mu-v)(d s d e)=0 \quad \forall t \in\right] 0, T\right], \text { a.s. },
$$

with

$$
H_{s}(e):=U_{s}(e)-\left(v\left(s, X_{s-}+\tilde{\gamma}(s, e)\right)-v\left(s, X_{s-}\right)\right) .
$$

If, in addition, $H \in \mathcal{G}_{\mathrm{loc}}^{2}(\mu)$, then there exists a predictable process $\left(l_{s}\right)$ such that

$$
H_{s}(e)=l_{s} \mathbb{1}_{K}(s), \quad d \mathbb{P} v(d s d e) \text {-a.e. }
$$

In particular,

$$
H_{S}(e)=0, \quad d \mathbb{P} v^{c}(d s d e) \text {-a.e. }
$$

and

$$
H_{S}(e)=l_{s} \mathbb{1}_{K}(s), \quad d \mathbb{P} v^{d}(d s d e) \text {-a.e. }
$$

The proof of Theorem 3.14 is based on the following stability result for càdlàg processes, which was the object of Proposition 5.37 in [5]. 
Proposition 3.15. Let $(X, Y)$ be a couple of $\left(\mathcal{F}_{t}\right)$-adapted processes satisfying Hypothesis 3.11 with corresponding function $v$. Then $v\left(t, X_{t}\right)$ is an $\left(\mathcal{F}_{t}\right)$-special weak Dirichlet process with decomposition

$$
\begin{aligned}
v\left(t, X_{t}\right)= & v\left(0, X_{0}\right)+\int_{] 0, t] \times \mathbb{R}}\left(v\left(s, X_{s-}+x\right)-v\left(s, X_{s-}\right)\right)\left(\mu^{X}-v^{X}\right)(d s d x) \\
& +A^{v}(t),
\end{aligned}
$$

where $A^{v}$ is a predictable $\left(\mathcal{F}_{t}\right)$-orthogonal process.

Proof of Theorem 3.14. By assumption, the couple $(X, Y)$ satisfies Hypothesis 3.11 with corresponding function $v$. We are then in the condition to apply Proposition 3.15 to $v\left(t, X_{t}\right)$, which gives (3.23). Set $\varphi(s, x):=v\left(s, X_{s-}+x\right)-v\left(s, X_{s-}\right)$. By condition (ii) in Hypothesis 3.11 , the process $\varphi$ verifies condition (2.18) with $A=\Omega \times[0, T] \times \mathbb{R}$. Moreover, $\varphi(s, 0)=0$. Since $\mu$ verifies Hypothesis 2.14, and $(X, \mu)$ verifies Hypothesis 2.9, we can apply Proposition 2.17 to $\varphi(s, x)$. Identity (3.23) becomes

$$
\begin{aligned}
v\left(t, X_{t}\right)= & v\left(0, X_{0}\right)+\int_{] 0, t] \times \mathbb{R}}\left(v\left(s, X_{s-}+\tilde{\gamma}(s, e)\right)-v\left(s, X_{s-}\right)\right)(\mu-v)(d s d e) \\
& +A^{v}(t) .
\end{aligned}
$$

At this point we recall that the process $Y_{t}=v\left(t, X_{t}\right)$ fulfills BSDE (3.2), which can be rewritten as

$$
Y_{t}=Y_{0}+\int_{] 0, t] \times \mathbb{R}} U_{s}(e)(\mu-v)(d s d e)-\int_{] 0, t] \times \mathbb{R}} \tilde{f}\left(s, e, Y_{s-}, U_{s}(e)\right) \lambda(d s d e) .
$$

The uniqueness of of the canonical decomposition (3.24) yields identity (3.19). If in addition we assume that $H \in \mathcal{G}_{\text {loc }}^{2}(\mu)$, the predictable bracket at time $t$ of the purely discontinuous martingale in identity (3.19) is well-defined, and equals $C(H)$ by Theorem 11.21, point (3), in [23]. Since $C(H)_{T}=0$ a.s., the conclusion follows from Proposition 2.8 .

\section{Applications}

\subsection{BSDEs driven by a jump-diffusion process}

Let us focus on the BSDE

$$
\begin{aligned}
Y_{t}= & g\left(X_{T}\right)+\int_{] t, T]} f\left(s, X_{s}, Y_{s}, Z_{s}, U_{s}(\cdot)\right) d s-\int_{] t, T]} Z_{s} d W_{s} \\
& -\int_{] t, T] \times \mathbb{R}} U_{s}(e)(\mu-v)(d s d e),
\end{aligned}
$$


which constitutes a particular case of the BSDE (1.1). This is considered for instance in [6]. Here $W$ is a Brownian motion and $\mu(d s d e)$ is a Poisson random measure with compensator

$$
v(d s d e)=\lambda(d e) d s
$$

where $\lambda$ is a Borel $\sigma$-finite measure on $\mathbb{R} \backslash\{0\}$ and

$$
\int_{\mathbb{R}}\left(1 \wedge|e|^{2}\right) \lambda(d e)<+\infty
$$

Poisson random measures have been introduced for instance, in Chapter II, Section 4.b in [27]. The process $X$ appearing in (4.1) is a Markov process satisfying the SDE

$$
d X_{s}=b\left(X_{s}\right) d s+\sigma\left(X_{s}\right) d W_{s}+\int_{\mathbb{R}} \gamma\left(X_{s-}, e\right)(\mu-v)(d s d e), \quad s \in[t, T],
$$

where $b: \mathbb{R} \rightarrow \mathbb{R}, \sigma: \mathbb{R} \rightarrow \mathbb{R}$ are globally Lipschitz, and $\gamma: \mathbb{R} \times \mathbb{R} \rightarrow \mathbb{R}$ is a measurable function such that, for some real $K$, and for all $e \in \mathbb{R}$,

$$
\begin{cases}|\gamma(x, e)| \leq K(1 \wedge|e|), & x \in \mathbb{R}, \\ \left|\gamma\left(x_{1}, e\right)-\gamma\left(x_{2}, e\right)\right| \leq K\left|x_{1}-x_{2}\right|(1 \wedge|e|), & x_{1}, x_{2} \in \mathbb{R} .\end{cases}
$$

For every starting point $x \in \mathbb{R}$ and initial time $t \in[0, T]$, there is a unique solution to (4.4) denoted $X^{t, x}$ (see [6], Section 1). Moreover, modulo suitable assumptions on the coefficients $(g, f)$, it is proved that the $\operatorname{BSDE}(4.1)$ admits a unique solution $(Y, Z, U) \in \mathcal{S}^{2} \times \mathcal{L}^{2} \times \mathcal{L}^{2}(\mu)$, see Theorem 2.1 in [6], where

$$
\begin{aligned}
& \mathcal{S}^{2}:=\left\{\left(Y_{t}\right)_{t \in[0, T]} \text { adapted càdlàg: }\left\|\sup _{t \in[0, T]}\left|Y_{t}\right|\right\|_{L^{2}(\Omega)}<\infty\right\}, \\
& \mathcal{L}^{2}:=\left\{\left(Z_{t}\right)_{t \in[0, T]} \text { predictable: } \mathbb{E}\left[\int_{0}^{T} Z_{s}^{2} d s\right]<\infty\right\},
\end{aligned}
$$

and $\mathcal{L}^{2}(\mu)$ is the space introduced in (2.5). When $X=X^{t, x}$, the solution $(Y, Z)$ of (4.1) is denoted $\left(Y^{t, x}, Z^{t, x}\right)$. In [6] it is proved that

$$
v(t, x):=Y_{t}^{t, x}, \quad(t, x) \in[0, T] \times \mathbb{R},
$$

satisfies $Y_{s}^{t, x}=v\left(s, X_{s}^{t, x}\right)$ for every $(t, x) \in[0, T] \times \mathbb{R}, s \in[t, T]$.

Lemma 4.1. Let $\mu$ and $X$ be respectively, the Poisson random measure and the stochastic process satisfying the SDE (4.4). Then $J=K=\varnothing, \mu$ satisfies Hypothesis 2.14 and $(X, \mu)$ fulfills Hypothesis 2.9 with

$$
X_{t}^{i}=\int_{] 0, t] \times \mathbb{R}} \gamma\left(X_{s-}, e\right)(\mu-v)(d s d e)
$$




$$
\begin{aligned}
X_{t}^{p} & =\int_{0}^{t} b\left(X_{s}\right) d s+\int_{0}^{t} \sigma\left(X_{s}\right) d W_{s}, \\
\tilde{\gamma}(\omega, s, e) & =\gamma\left(X_{s-}(\omega), e\right) .
\end{aligned}
$$

Proof. Our aim is to apply Lemma 2.19. We start by noticing that $v$ in (4.2) is in the form (2.1) with $A_{s}=s$. Therefore by Remark 2.16 item 2 . and successively item $1 ., J=K=\varnothing$, and Hypothesis 2.14 is verified. On the other hand, the process $X$ satisfies the stochastic differential equation (4.4), which is a particular case of (2.20) when $B_{s}=s, N_{s}=W_{s}$, and $b, \sigma, \gamma$ are time homogeneous. $b$ and $\sigma$ verify (2.21), (2.22) since they have linear growth. Condition (2.23) can be verified using the characterization of $\mathcal{G}_{\text {loc }}^{1}(\mu)$ in Theorem 1.33, point (c), Chapter II, in [27]: for a given predictable random field $W$ defined on $\tilde{\Omega}$ such that $\hat{W}=0$, that theorem specifies that, whenever $|W|^{2} \mathbb{1}_{\{|W| \leq 1\}} \star v+|W| \mathbb{1}_{\{|W|>1\}} \star v \in \mathcal{A}_{\text {loc }}^{+}$, then $W \in \mathcal{G}_{\text {loc }}^{1}(\mu)$. That property follows from (4.3) and (4.5).

Then, by Lemma 2.19, $X$ verifies Hypothesis 2.9 with decomposition $X=X^{i}+X^{p}$, where $X^{i}$ and $X^{p}$ are given respectively by (4.7) and (4.8), and with $\tilde{\gamma}(\omega, s, e)=\gamma\left(X_{s-}(\omega), e\right)$.

We aim at applying Theorem 3.14 to BSDE (4.1). To this end, we need the following preliminary result.

Lemma 4.2. Let $\mu$ and $X$ be respectively the Poisson random measure and the stochastic process satisfying the $S D E$ (4.4). Let $v:[0, T] \times \mathbb{R} \rightarrow \mathbb{R}$ be a function of $C^{0,1}$ class such that $x \mapsto$ $\partial_{x} v(s, x)$ has linear growth, uniformly in $s$. Then condition (3.6) holds for $X$ and $v$.

Proof. We have

$$
\begin{aligned}
\int_{] 0, \cdot] \times \mathbb{R}}\left|v\left(s, X_{s-}+x\right)-v\left(s, X_{s-}\right)-x \partial_{x} v\left(s, X_{s-}\right)\right| \mathbb{1}_{\{|x|>1\}} \mu^{X}(d s d x) \\
\quad=\sum_{0<s \leq \cdot}\left|v\left(s, X_{s}\right)-v\left(s, X_{s-}\right)-\partial_{x} v\left(s, X_{s-}\right) \Delta X_{s}\right| \mathbb{1}_{\left\{\left|\Delta X_{s}\right|>1\right\}} \\
\quad \leq \sum_{0<s \leq \cdot}\left|\Delta X_{s}\right| \mathbb{1}_{\left\{\left|\Delta X_{s}\right|>1\right\}}\left(\int_{0}^{1}\left|\partial_{x} v\left(s, X_{s-}+a \Delta X_{s}\right)\right| d a+\int_{0}^{1}\left|\partial_{x} v\left(s, X_{s-}\right)\right| d a\right) \\
\leq 2 C \sum_{0<s \leq \cdot}\left|X_{s-}\right|\left|\Delta X_{s}\right| \mathbb{1}_{\left\{\left|\Delta X_{s}\right|>1\right\}}+\sum_{s \leq t}\left|\Delta X_{s}\right|^{2} C \mathbb{1}_{\left\{\left|\Delta X_{s}\right|>1\right\}} \\
\quad=2 C \int_{j 0, \cdot] \times \mathbb{R}}\left|X_{s-}\right||x| \mathbb{1}_{\{|x|>1\}} \mu^{X}(d s d x)+\sum_{s \leq \cdot}\left|\Delta X_{s}\right|^{2} \mathbb{1}_{\left\{\left|\Delta X_{s}\right|>1\right\}},
\end{aligned}
$$

for some constant $C$. Since $X$ is of finite quadratic variation, by Lemma 2.10(ii) in [5] we have that $\sum_{s \in] 0, T]}\left|\Delta X_{S}\right|^{2}<\infty$, a.s. Consequently, the second term in the right-hand side of (4.9) belongs to $\mathcal{A}_{\text {loc }}^{+}$if we prove that

$$
\sum_{s \in] 0, \cdot]}\left|\Delta X_{s}\right|^{2} \in \mathcal{A}_{\mathrm{loc}}^{+} .
$$


Since by (4.4) $\Delta X_{s}=\int_{\mathbb{R}} \gamma\left(X_{s-}, e\right) \mu(d s d e)$, we have

$$
\sum_{s \in] 0, \cdot]}\left|\Delta X_{s}\right|^{2}=\sum_{s \in] 0, \cdot]}\left|\int_{\mathbb{R}} \gamma\left(X_{s-}, e\right) \mu(d s d e)\right|^{2}=\int_{] 0, \cdot] \times \mathbb{R}}\left|\gamma\left(X_{s-}, e\right)\right|^{2} \mu(d s d e),
$$

and (4.10) reads

$$
\int_{] 0, \cdot] \times \mathbb{R}}\left|\gamma\left(X_{s-}, e\right)\right|^{2} \mu(d s d e) \in \mathcal{A}_{\mathrm{loc}}^{+} .
$$

The integral in the left-hand side of (4.11) exists almost surely. Indeed, $|\gamma(x, e)| \leq K(1 \wedge|e|)$ for every $x \in \mathbb{R}, \int_{\mathbb{R}}\left(1 \wedge|e|^{2}\right) \lambda(d e)<\infty$ (see, respectively, (4.5) and (4.3)). Since it is càglàd, then it is locally bounded, see for instance the lines above Theorem 15, Chapter IV, in [36]. Consequently, it belongs to $\mathcal{A}_{\text {loc }}^{+}$.

Finally, the first term in the right-hand side of (4.9) belongs to $\mathcal{A}_{\text {loc }}^{+}$, taking into account (3.5) and the fact that $X_{s-}$ is locally bounded being càglàd. The conclusion follows.

We are ready to give the identification result.

Corollary 4.3. Let $(Y, Z, U) \in \mathcal{S}^{2} \times \mathcal{L}^{2} \times \mathcal{L}^{2}(\mu)$ be the unique solution to the BSDE (4.1). If the function $v$ defined in (4.6) is of class $C^{0,1}$ such that $x \mapsto \partial_{x} v(t, x)$ has linear growth, uniformly in $t$, then the pair $(Z, U)$ satisfies

$$
\begin{gathered}
Z_{t}=\sigma\left(X_{t}\right) \partial_{x} u\left(t, X_{t}\right) \quad d \mathbb{P} d t \text {-a.e., } \\
\left.\left.\int_{] 0, t] \times \mathbb{R}} H_{S}(e)(\mu-v)(d s d e)=0, \quad \forall t \in\right] 0, T\right], \text { a.s., }
\end{gathered}
$$

where

$$
H_{s}(e):=U_{s}(e)-\left(v\left(s, X_{s-}+\gamma\left(s, X_{s-}, e\right)\right)-v\left(s, X_{s-}\right)\right) .
$$

If in addition $H \in \mathcal{G}_{\mathrm{loc}}^{2}(\mu)$,

$$
U_{s}(e)=v\left(s, X_{s-}+\gamma\left(s, X_{s-}, e\right)\right)-v\left(s, X_{s-}\right) \quad d \mathbb{P} \lambda(d e) d s \text {-a.e. }
$$

Proof. We aim at applying Theorem 3.8. By Lemma 4.1, $\mu$ satisfies Hypothesis 2.14 with $\tilde{\gamma}(s, e)=\gamma\left(s, X_{s-}, e\right)$. Since $X$ is a special semimartingale, then condition (3.5) holds by Corollary 11.26 in [23]. Moreover, $X$ is obviously a special weak Dirichlet process with finite quadratic variation. By Lemma 4.2, condition (3.6) holds for $X$ and $v$, which implies that Hypothesis 3.7 is verified.

We can then apply Theorem 3.8: since $X^{c}=\int_{0}^{\cdot} \sigma\left(X_{t}\right) d W_{t}$ and $M=W$, (3.9) gives (4.12), while (3.10)-(3.11) with $\tilde{\gamma}(s, e)=\gamma\left(s, X_{s-}, e\right)$ yield (4.13)-(4.14). If in addition $H \in \mathcal{G}^{2}(\mu)$, since $J=K=\varnothing$, see Lemma 4.1, $v=v^{c}$ and (4.15) follows by (3.12). 
Remark 4.4. When the BSDE (4.1) is driven only by a standard Brownian motion, an identification result for $Z$ analogous to (4.12) has been established by [20], even supposing only that $f$ is Lipschitz with respect to $Z$.

Remark 4.5. Theorem 3.8 also potentially applies to the cases of BSDEs driven by a continuous martingale when the underlying process $X$ is a solution of an SDE with singular (distributional) drift. In the literature, there are plenty of cases of (even continuous) Markov processes that are not semimartingales. Typical examples of such underlying processes $X$ are solutions of an SDE with distributional drift, see, for example, [18,19,37], of the type

$$
d X_{t}=\beta\left(X_{t}\right) d t+d W_{t}
$$

for a class of Schwartz distributions $\beta$. In this case, $X$ is generally not a semimartingale but only a Dirichlet process, so that, for $v \in C^{0,1}, v\left(t, X_{t}\right)$ is a (special) weak Dirichlet process. Forward BSDEs related to an underlying process $X$ solving (4.16) have been studied for instance in [38], when the terminal type is random. However we do not perform a more refined analysis of examples in that direction since it goes beyond the scope of the paper.

\subsection{On a class of BSDEs driven by a quasi-left-continuous random measure}

In [13], the authors study a BSDE driven by an integer-valued random measure $\mu$ associated to a given pure jump Markov process $X$, of the form

$$
Y_{t}=g\left(X_{T}\right)+\int_{] t, T]} f\left(s, X_{s}, Y_{s}, U_{s}(\cdot)\right) d s-\int_{] t, T] \times \mathbb{R}} U_{s}(e)(\mu-v)(d s d e) .
$$

The underlying process $X$ is generated by a marked point process $\left(T_{n}, \zeta_{n}\right)$, where $\left(T_{n}\right)_{n}$ are increasing random times such that $\left.T_{n} \in\right] 0, \infty[$, where either there is a finite number of times $\left(T_{n}\right)_{n}$ or $\lim _{n \rightarrow \infty} T_{n}=+\infty$, and $\zeta_{n}$ are random variables in $\mathbb{R}$, see, for example, Chapter III, Section $2 \mathrm{~b}$., in [25]. This means that $X$ is a càdlàg process such that $X_{t}=\zeta_{n}$ for $t \in\left[T_{n}, T_{n+1}[\right.$, for every $n \in \mathbb{N}$. In particular, $X$ has a finite number of jumps on each compact. The associated integer-valued random measure $\mu$ is the sum of the Dirac measures concentrated at the marked point process $\left(T_{n}, \zeta_{n}\right)$, and can be written as

$$
\mu(d s d e)=\sum_{s \in[0, T]} \mathbb{1}_{\left\{X_{s-} \neq X_{s}\right\}} \delta_{\left(s, X_{s}\right)}(d t d e)
$$

Given a measure $\mu$ in the form (4.18), it is related to the jump measure $\mu^{X}$ in the following way: for every Borel subset $A$ of $\mathbb{R}$,

$$
\int_{] 0, T] \times \mathbb{R}} \mathbb{1}_{A}\left(e-X_{s-}\right) \mu(d s d e)=\int_{] 0, T] \times \mathbb{R}} \mathbb{1}_{A}(x) \mu^{X}(d s d x) .
$$


This is for instance explained in Example 3.22 in [25]. The pure jump process $X$ then satisfies the equation

$$
X_{t}=X_{0}+\sum_{0<s \leq t} \Delta X_{s}=X_{0}+\int_{] 0, t] \times \mathbb{R}}\left(e-X_{s-}\right) \mu(d s d e) .
$$

The compensator of $\mu(d s d e)$ is

$$
v(d s d e)=\lambda\left(s, X_{s-}, d e\right) d s,
$$

where $\lambda$ the is the transition rate measure of the process satisfying

$$
\sup _{t \in[0, T], x \in \mathbb{R}} \lambda(t, x, \mathbb{R})<\infty,
$$

see Section 2.1 in [13].

Under suitable assumptions on the coefficients $(g, f)$, Theorem 3.4 in [13] states that the $\operatorname{BSDE}(4.17)$ admits a unique solution $(Y, U) \in \mathcal{L}^{2} \times \mathcal{L}^{2}(\mu)$, where $\mathcal{L}^{2}(\mu)$ and $\mathcal{L}^{2}$ are the spaces introduced in Section 4.1. Theorem 4.4 in [13] shows moreover that there exists a measurable function $v:[0, T] \times \mathbb{R} \rightarrow \mathbb{R}$ such that

$$
\begin{aligned}
& \forall e \in \mathbb{R}, t \mapsto v(t, e) \quad \text { is absolutely continuous on }[0, T], \\
& \left(v\left(s, X_{s}\right)\right) \in \mathcal{L}^{2} \quad \text { and } \quad\left(v(s, e)-v\left(s, X_{s-}\right),(s, e) \in[0, T] \times \mathbb{R}\right) \in \mathcal{L}^{2}(\mu),
\end{aligned}
$$

and the unique solution of the BSDE (4.17) can be represented as

$$
Y_{s}=v\left(s, X_{s}\right), \quad s \in[0, T] .
$$

Lemma 4.6. Let $\mu$ be the integer-valued random measure in (4.18) with compensator $v$ given by (4.21), and $X$ be the associated pure jump Markov process satisfying (4.20). Then $J=K=\varnothing$, $\mu$ satisfies Hypothesis 2.14 and $(X, \mu)$ fulfills Hypothesis 2.9 with $\tilde{\gamma}(\omega, s, e)=e-X_{s-}(\omega)$.

Proof. Since $v$ in (4.21) is in the form (2.1) with $A_{s}=s$, by Remark 2.16, item 2. and successively item $1 ., J=K=\varnothing$ and Hypothesis 2.14 is verified. Let us now prove that $(X, \mu)$ fulfills Hypothesis 2.9 with $\tilde{\gamma}(\omega, s, e)=e-X_{s-}(\omega)$. We observe that (4.18) implies that $\{\Delta X \neq 0\}=D$. Therefore, recalling that by Remark 2.16-1., $D=\bigcup_{n}\left[\left[T_{n}^{i}\right]\right]$ for some sequence of totally inaccessible times $\left(T_{n}^{i}\right)_{n}$ such that $\left[\left[T_{n}^{i}\right]\right] \cap\left[\left[T_{m}^{i}\right]\right]=\varnothing, n \neq m$, we see that $X=X^{i}$ is quasi-left-continuous.

Finally, by definition of $\mu$ we have

$$
\mathbb{E}\left[\int_{] 0, T] \times \mathbb{R}} \mu(d s d e)\left|\left(e-X_{s-}\right)-\Delta X_{s}\right|\right]=0,
$$

therefore $X^{i}$ satisfies Hypothesis 2.9-1. with $\tilde{\gamma}(\omega, s, e)=e-X_{s-}(\omega)$. Since $X^{p}=0$, Hypothesis 2.9-2. trivially holds. 
Let us now apply Theorem 3.14 to the present framework. We start with a preliminary observation.

Lemma 4.7. Let $\mu$ be the integer-valued random measure in (4.18) with compensator $v$ given by (4.21), and $X$ be the associated pure jump Markov process satisfying (4.20). Let $v:[0, T] \times \mathbb{R} \rightarrow$ $\mathbb{R}$ be a function satisfying (4.24) and such that, for $E=\mathbb{R}$,

$$
\forall e \in E, t \mapsto v(t, e) \quad \text { is continuous on }[0, T] .
$$

Suppose that $Y_{t}=v\left(t, X_{t}\right)$ is an $\left(\mathcal{F}_{t}\right)$-orthogonal process. Then $(X, Y)$ satisfies Hypothesis 3.11 with corresponding function $v$.

Proof. By Remark 3.12, from (4.26) it follows that Hypothesis 3.11(iii) is verified. Taking into account Remark 3.13, it follows that $Y_{t}=v\left(t, X_{t}\right)$ has a finite number of jumps, so that $\sum_{s \leq T}\left|\Delta Y_{s}\right|<\infty$ a.s. In particular, Hypothesis 3.11(i) holds.

To verify the validity of condition (ii) of Hypothesis 3.11, we have to show that (3.18) holds. Denoting $\|\lambda\|_{\infty}=\sup _{t \in[0, T], x \in \mathbb{R}}|\lambda(t, x, \mathbb{R})|$, by (4.19) we have

$$
\begin{aligned}
& \mathbb{E}\left[\int_{] 0, T] \times \mathbb{R}}\left|v\left(s, X_{s-}+x\right)-v\left(s, X_{s-}\right)\right| \mu^{X}(d s d x)\right] \\
& \quad=\mathbb{E}\left[\int_{] 0, T] \times \mathbb{R}}\left|v(s, e)-v\left(s, X_{s-}\right)\right| \mu(d s d e)\right] \\
& \quad=\mathbb{E}\left[\int_{] 0, T] \times \mathbb{R}}\left|v(s, e)-v\left(s, X_{s-}\right)\right| \lambda\left(s, X_{s-}, d e\right) d s\right] \\
& \quad \leq T\|\lambda\|_{\infty}^{1 / 2}\left\|v(s, e)-v\left(s, X_{s-}\right)\right\|_{\mathcal{L}^{2}(\mu)}^{1 / 2}
\end{aligned}
$$

and the conclusion follows since $v(s, e)-v\left(s, X_{s-}\right) \in \mathcal{L}^{2}(\mu)$ by (4.24).

We have the following identification result.

Corollary 4.8. Let $(Y, U) \in \mathcal{L}^{2} \times \mathcal{L}^{2}(\mu)$ be the unique solution to the BSDE (4.17) and $X, v$ be respectively, the process and the function appearing in (4.25). Then the random field $U$ satisfies

$$
U_{t}(e)=v(t, e)-v\left(t, X_{t-}\right) \quad d \mathbb{P} \lambda\left(t, X_{t-}, d e\right) d t \text {-a.e. }
$$

Proof. We aim at applying Theorem 3.14. By Lemma 4.6, $\mu$ satisfies Hypothesis 2.14 and $(X, \mu)$ fulfills Hypothesis 2.9 with $\tilde{\gamma}(s, e)=e-X_{s-}$. Moreover, by Lemma 4.7 and Remark 3.3, $(X, Y)$ satisfies Hypothesis 3.11 with corresponding function $v$. We can then apply Theorem 3.14. We have

$$
\begin{aligned}
H_{S}(e) & :=U_{s}(e)-\left(v\left(s, X_{s-}+\tilde{\gamma}(s, e)\right)-v\left(s, X_{s-}\right)\right) \\
& =U_{s}(e)-\left(v(s, e)-v\left(s, X_{s-}\right)\right),
\end{aligned}
$$


which belongs to $\mathcal{L}^{2}(\mu)$, and therefore to $\mathcal{G}^{2}(\mu)$. Recalling that $J=K=\varnothing$, see Lemma 4.6, $v=v^{c}$ and (4.27) follows by (3.21).

Remark 4.9. The result in Corollary 4.8 is not new, since it retrieves with a different method, without using the specific form of $v$, the result obtained in [13]. In particular, our identification does not need to use the absolute continuity property (4.23).

\subsection{On a class of BSDEs driven by a non quasi-left-continuous random measures}

In the recent paper [2], the author studies the existence and uniqueness for a BSDE driven by a purely discontinuous martingale of the form

$$
Y_{t}=\xi+\int_{] t, T]} \tilde{f}\left(s, Y_{s-}, U_{s}(\cdot)\right) d A_{s}-\int_{] t, T] \times \mathbb{R}} U_{s}(e)(\mu-v)(d s d e),
$$

for given data $\xi, \tilde{f}$. Here $\mu(d s d e)$ is an integer-valued random measure with compensator $v(d s d e)=d A_{s} \phi_{s}(d e)$, where $\phi$ is a probability kernel and $A$ is a right-continuous nondecreasing predictable process, such that $\hat{v}_{s}(\mathbb{R})=\Delta A_{s} \leq 1$ for every $s$. For any positive constant $\beta, \mathcal{E}^{\beta}$ will denote the Doléans-Dade exponential of the process $\beta A$. We consider the weighted spaces

$$
\begin{aligned}
\mathcal{L}_{\beta}^{2}(A):= & \left\{\text { adapted càdl àg processes }\left(Y_{s}\right)_{s \in[0, T]}, \text { s.t. } \mathbb{E}\left[\int_{0}^{T} \mathcal{E}_{s}^{\beta}\left|Y_{s-}\right|^{2} d A_{s}\right]<\infty\right\}, \\
\mathcal{G}_{\beta}^{2}(\mu):= & \left\{\text { predictable processes }\left(U_{s}(\cdot)\right)_{s \in[0, T]},\right. \text { s.t. } \\
& \|U\|_{\mathcal{G}_{\beta}^{2}(\mu)}^{2}:=\mathbb{E}\left[\int_{] 0, T] \times \mathbb{R}} \mathcal{E}_{s}^{\beta}\left|U_{s}(e)-\hat{U}_{s}\right|^{2} v(d s d e)+\sum_{s \in] 0, T]} \mathcal{E}_{s}^{\beta}\left|\hat{U}_{s}\right|^{2}\left(1-\Delta A_{s}\right)\right] \\
& <\infty\} .
\end{aligned}
$$

In [2], the author considers solutions $(Y, U) \in \mathcal{L}_{\beta}^{2}(A) \times \mathcal{G}_{\beta}^{2}(\mu)$. Suitable assumptions are required on the triplet $(\tilde{f}, \xi, \beta)$. In particular $\tilde{f}$ is of Lipschitz type in the third and fourth variable and $\xi$ is a square integrable random variable with some weight. Moreover, the following technical assumption has to be fulfilled: there exists $\varepsilon \in] 0,1[$ such that

$$
2\left|L_{y}\right|^{2}\left|\Delta A_{t}\right|^{2} \leq 1-\varepsilon, \quad \mathbb{P} \text {-a.s., } \forall t \in[0, T],
$$

where $L_{y}$ is the Lipschitz constant of $\tilde{f}$ with respect to $y$. Under these hypotheses, for $\beta$ large enough, it can be proved that there exists a (unique) solution $(Y, U) \in \mathcal{L}_{\beta}^{2}(A) \times \mathcal{G}_{\beta}^{2}(\mu)$ to BSDE (4.29), see Theorem 4.1 in [2]. In that theorem, one shows that, given two solutions $(Y, U)$, $\left(Y^{\prime}, U^{\prime}\right)$, then we have $Y_{t}=Y_{t}^{\prime} d \mathbb{P} d A_{t}$-a.e. and $\left\|U-U^{\prime}\right\|_{\mathcal{G}_{\beta}^{2}(\mu)}^{2}=0$. This implies that $\| U-$ 
$U^{\prime} \|_{\mathcal{G}^{2}(\mu)}^{2}=0$, and so, by Remark 3.1, there is a predictable process $\left(l_{t}\right)$ such that $U_{t}(e)-U_{t}^{\prime}(e)=$ $l_{t} \mathbb{1}_{K}(t), d \mathbb{P} v(d t d e)$-a.e.

\subsubsection{The PDMPs case}

Let us now consider a particular case of BSDE (4.29), namely a BSDE driven by the integervalued random measure $\mu$ associated to a given Markov process $X$, of the form

$$
Y_{t}=g\left(X_{T}\right)+\int_{] t, T]} f\left(s, X_{s-}, Y_{s-}, U_{s}(\cdot)\right) d A_{s}-\int_{] t, T] \times \mathbb{R}} U_{s}(e)(\mu-v)(d s d e) .
$$

We assume that $X$ is a piecewise deterministic Markov process (PDMP) associated to a random measure $\mu$, with values in the interval ]0, 1[. The process $X$ is generated by a marked point process $\left(T_{n}, \zeta_{n}\right)$, where $\left(T_{n}\right)_{n}$ are increasing random times such that $\left.T_{n} \in\right] 0, \infty[$, where either there is a finite number of times $\left(T_{n}\right)_{n}$ or $\lim _{n \rightarrow \infty} T_{n}=+\infty$, and $\zeta_{n}$ are random variables in ]0, 1[.

We will follow the notations in [15], Chapter 2, Sections 24 and 26. The behavior of the PDMP $X$ is described by a triplet of local characteristics $(h, \lambda, P): h:] 0,1[\rightarrow \mathbb{R}$ is a Lipschitz continuous function, $\lambda:] 0,1[\rightarrow \mathbb{R}$ is a measurable function satisfying

$$
\sup _{x \in] 0,1[}|\lambda(x)|<\infty
$$

and $P$ is a transition probability measure on $[0,1] \times \mathcal{B}(] 0,1[)$. Some other technical assumptions are specified in the over-mentioned reference, that we not recall here. Let us denote by $\Phi(s, x)$ the unique solution of $g^{\prime}(s)=h(g(s)), g(0)=x$. The process $X$ can be defined as

$$
X(t)= \begin{cases}\Phi(t, x), & t \in\left[0, T_{1}[\right. \\ \Phi\left(t-T_{n}, \zeta_{n}\right), & t \in\left[T_{n}, T_{n+1}[.\right.\end{cases}
$$

Set $N_{t}=\sum_{n \in \mathbb{N}} \mathbb{1}_{t \geq T_{n}}$. By Proposition 24.6 in [15], we have

$$
\mathbb{E}\left[N_{t}\right]<\infty, \quad \forall t \in \mathbb{R}_{+}
$$

Notice that the PDMP $X$ verifies the equation

$$
X_{t}=X_{0}+\int_{0}^{t} h\left(X_{s}\right) d s+\sum_{0<s \leq t} \Delta X_{s} .
$$

In particular, $X$ admits a finite number of jumps on each compact interval. By (26.9) in [15], the random measure $\mu$ is

$$
\mu(d s d e)=\sum_{n} \mathbb{1}_{\left\{\zeta_{n} \in\right] 0,1[\}} \delta_{\left(T_{n}, \zeta_{n}\right)}(d s d e)=\sum_{0<s \leq t} \mathbb{1}_{\left\{X_{s-} \neq X_{s}\right\}} \delta_{\left(s, X_{s}\right)}(d s d e),
$$


which is of the type of (4.18). This implies the validity of (4.19), so that (4.35) can be rewritten as

$$
X_{t}=X_{0}+\int_{0}^{t} h\left(X_{s}\right) d s+\int_{] 0, t] \times] 0,1[}\left(e-X_{s-}\right) \mu(d s d e) .
$$

In the following, by abuse of notations, $\mu$ will denote the trivial extension of previous measure to the real line in the space variable $e$. In particular (4.35) can be reexpressed as

$$
X_{t}=X_{0}+\int_{0}^{t} h\left(X_{s}\right) d s+\int_{] 0, t] \times \mathbb{R}}\left(e-X_{s-}\right) \mu(d s d e) .
$$

The knowledge of $(h, \lambda, P)$ completely specifies the dynamics of $X$, see Section 24 in [15]. According to (26.2) in [15], the compensator of $\mu$ has the form

$$
v(d s d e)=\left(\lambda\left(X_{s-}\right) d s+d p_{s}^{*}\right) P\left(X_{s-}, d e\right),
$$

where $\lambda$ has been trivially extended to $[0,1]$ by the zero value, and

$$
p_{t}^{*}=\sum_{n=1}^{\infty} \mathbb{1}_{\left\{t \geq T_{n}\right\}} \mathbb{1}_{\left\{X_{T_{n}-} \in\{0,1\}\right\}}
$$

is the predictable process counting the number of jumps of $X$ from the boundary of its domain.

From (4.38), we can choose $A_{s}$ and $\phi_{s}(d e)$ such that $d A_{s}=\lambda\left(X_{s-}\right) d s+d p_{s}^{*}$ and $\phi_{s}(d e)=$ $P\left(X_{s-}, d e\right)$. In particular, $A$ is predictable (not deterministic) and discontinuous, with jumps

$$
\Delta A_{s}(\omega)=\hat{v}_{s}(\omega, \mathbb{R})=\Delta p_{s}^{*}(\omega)=\mathbb{1}_{\left\{X_{s-}(\omega) \in\{0,1\}\right\}} .
$$

Consequently, $\hat{v}_{t}(\omega, \mathbb{R})>0$ if and only if $\hat{v}_{t}(\omega, \mathbb{R})=1$, so that

$$
J=\left\{(\omega, t): \hat{v}_{t}(\omega, \mathbb{R})>0\right\}=\left\{(\omega, t): \hat{v}_{t}(\omega, \mathbb{R})=1\right\}=K,
$$

and

$$
K=\left\{(\omega, t): X_{t-}(\omega) \in\{0,1\}\right\} .
$$

Lemma 4.10. Let $X$ be the PDMP process with local characteristics $(h, \lambda, P)$, satisfying (4.35). Then

$$
\int_{\mathrm{j} 0, \cdot] \times \mathbb{R}}\left|e-X_{s-}\right| v(d s d e) \in \mathcal{A}_{\mathrm{loc}}^{+} .
$$

Proof. We start by noticing that $\int_{] 0, T] \times \mathbb{R}}\left|e-X_{s-}\right| v(d s d e)<\infty$, a.s. Indeed

$$
\begin{aligned}
\int_{] 0, T] \times \mathbb{R}}\left|e-X_{s-}\right| v(d s d e) & =\int_{] 0, T] \times] 0,1[}\left|e-X_{s-}\right|\left(\lambda\left(X_{s-}\right) d s+d p_{s}^{*}\right) P\left(X_{s-}, d e\right) \\
& \leq\|\lambda\|_{\infty}\left(T+p_{T}^{*}\right) .
\end{aligned}
$$


For every $t \in[0, T]$, the jumps of the process $\Gamma_{t}:=\int_{] 0, t] \times \mathbb{R}}\left|e-X_{s-}\right| v(d s d e)$ are given by

$$
\Delta \Gamma_{t}:=\int_{] 0,1[}\left|e-X_{t-}\right| \hat{v}_{t}(d e) \leq \hat{v}_{t}(\mathbb{R}) \leq 1 .
$$

Since $\Gamma_{t}$ has bounded jumps, it is a locally bounded process and therefore it belongs to $\mathcal{A}_{\text {loc }}^{+}$, see for instance the proof of Corollary at page 373 in [36].

Lemma 4.11. Let $\mu$ and $X$ be respectively, the random measure and the associated PDMP with local characteristics $(h, \lambda, P)$ satisfying equation (4.37). Assume in addition that there exists a function $\beta:\{0,1\} \rightarrow] 0,1[$, such that

$$
P(x, d e)=\delta_{\beta(x)}(d e) \quad \text { a.s. }
$$

Then $\mu$ satisfies Hypothesis 2.14 and $(X, \mu)$ fulfills Hypothesis 2.9 with

$$
\begin{aligned}
X_{t}^{i}= & \int_{] 0, t] \times \mathbb{R}}\left(e-X_{s-}\right)(\mu-v)(d s d e), \\
X_{t}^{p}= & X_{0}+\int_{0}^{t} h\left(X_{s}\right) d s \\
& +\int_{] 0, t]}\left(\int_{\mathbb{R}}\left(e-X_{s-}\right) P\left(X_{s-}, d e\right)\right)\left(\lambda\left(X_{s-}\right) d s+d p_{s}^{*}\right), \\
\tilde{\gamma}(\omega, s, e)= & \left(e-X_{s-}(\omega)\right) \mathbb{1}_{\left\{X_{s-}(\omega) \in\right] 0,1[\}}(\omega, s) .
\end{aligned}
$$

Remark 4.12. Condition (4.43) implies that

$$
X_{s}=\beta\left(X_{s-}\right) \quad \text { on }\left\{(\omega, s): X_{s-}(\omega) \in\{0,1\}\right\}
$$

Proof. Let us prove that Hypothesis 2.14(i) holds. We recall that the measure $\mu$ was characterized by (4.36). Moreover, given $\mu^{c}=\mu \mathbb{1}_{J^{c}}$ and $v^{c}=v \mathbb{1}_{J^{c}}, v^{c}$ is the compensator of $\mu^{c}$, see paragraph (b) in [24]. Taking into account (4.38), (4.40) and (4.41), we have

$$
v^{c}(d s d e)=\lambda\left(X_{s-}\right) P\left(X_{s-}, d e\right) d s .
$$

We remark that $D \cap J^{c}=\left\{(\omega, t): \mu^{c}(\omega,\{t\} \times \mathbb{R})>0\right\}$. By Remark 2.2(ii), we have $D \cap J^{c}=$ $\bigcup_{n}\left[\left[T_{n}^{i}\right]\right],\left(T_{n}^{i}\right)_{n}$ totally inaccessible times. On the other hand, since by (4.41) $J=K$, we have $D=K \cup\left(D \cap J^{c}\right)$, therefore Hypothesis 2.14(i) holds.

Let us now consider Hypothesis 2.14(ii). Taking into account (4.42), we have to prove that for every predictable time $S$ such that $[[S]] \subset\left\{(\omega, t): X_{t-}(\omega) \in\{0,1\}\right\}$,

$$
v(\{S\}, d e)=\mu(\{S\}, d e) \quad \text { a.s. }
$$

Let $S$ be a predictable time satisfying $[[S]] \subset\left\{(\omega, t): X_{t-}(\omega) \in\{0,1\}\right\}$. By $(4.36), \mu(\{S\}, d e)=$ $\delta_{X_{S}}(d e)$, while from (4.38) we get $v(\{S\}, d e)=P\left(X_{S-}, d e\right)$. Therefore, identity (4.48) can be 
rewritten as

$$
P\left(X_{S_{-}}, d e\right)=\delta_{X_{S}}(d e) \quad \text { a.s. }
$$

Previous identity holds true under assumptions (4.46) and (4.43), and so Hypothesis 2.14(ii) is established.

In order to prove the validity of Hypothesis 2.9, we will make use of Lemma 2.19. We recall that the process $X$ satisfies the stochastic differential equation (4.37), which gives, taking into account (4.38) and Lemma 4.10,

$$
\begin{aligned}
X_{t}= & X_{0}+\int_{0}^{t} h\left(X_{s}\right) d s+\int_{] 0, t]}\left(\int_{\mathbb{R}}\left(e-X_{s}\right) P\left(X_{s}, d e\right)\right) \lambda\left(X_{s}\right) d s \\
& +\int_{] 0, t]}\left(\beta\left(X_{s-}\right)-X_{s-}\right) d p_{s}^{*}+\int_{] 0, t] \times \mathbb{R}}\left(e-X_{s-}\right)(\mu-v)(d s d e) .
\end{aligned}
$$

We can show that previous equation is a particular case of (2.20). Indeed, we recall that, by (4.39) and (4.42), the support of the measure $d p^{*}$ is included in $K$. We set $B_{s}=s+p^{*}(s)$ and $b(s, x)=\left(h(x)+\int_{\mathbb{R}}(e-x) \lambda(x) P(x, d e)\right) \mathbb{1}_{K^{c}}(s)+(\beta(x)-x) \mathbb{1}_{K}(s)$. The reader can easily show that the sum of the first, second, and third integral in the right-hand side of (4.50) equals $\int_{0}^{t} b\left(s, X_{s-}\right) d B_{s}$, provided we show that $\int_{0}^{T}\left|b\left(s, X_{s-}\right)\right| d B_{s}$ is finite a.s. In fact we have

$$
\begin{aligned}
& \int_{0}^{t}\left|b\left(s, X_{s-}\right)\right| d B_{s} \\
& \leq \int_{0}^{t}\left|h\left(X_{s}\right)\right| d s \\
& \quad+\int_{] 0, t]}\left|\int_{\mathbb{R}}\left(e-X_{s-}\right) \lambda\left(X_{s-}\right) P\left(X_{s-}, d e\right) \mathbb{1}_{K^{c}}(s)+\left(\beta\left(X_{s-}\right)-X_{s-}\right) \mathbb{1}_{K}(s)\right| d B_{s} \\
& =\int_{0}^{t}\left|h\left(X_{S}\right)\right| d s \\
& \quad+\int_{] 0, t]}\left|\int_{\mathbb{R}}\left(e-X_{s-}\right) P\left(X_{s-}, d e\right)\left(\lambda\left(X_{s-}\right) \mathbb{1}_{K^{c}}(s)+\mathbb{1}_{K}(s)\right)\right|\left(d s+d p^{*}(s)\right) \\
& \leq \int_{0}^{t}\left|h\left(X_{S}\right)\right| d s+\int_{10, t]} \int_{\mathbb{R}}\left|e-X_{s-}\right| v(d s, d e) .
\end{aligned}
$$

Recalling Lemma 4.10, and taking into account that $h$ is locally bounded, we get that $\int_{0}^{\cdot}\left|b\left(s, X_{s-}\right)\right| d B_{s}$ belongs to $\mathcal{A}_{\mathrm{loc}}^{+}$. Then, setting $N_{s}=0$ and $\gamma(s, x, e)=e-x$, we see that $X$ is a solution to equation (2.20).

Then, by Lemma 2.19, $(X, \mu)$ satisfies Hypothesis 2.9 with decomposition $X=X^{i}+$ $X^{p}$, where $X^{i}$ and $X^{p}$ are given respectively, by (4.44) and (4.45). In particular, the process $X^{i}$ fulfills Hypothesis 2.9-1. with $\tilde{\gamma}(\omega, s, e)=\left(e-X_{s-}(\omega)\right)\left(1-\mathbb{1}_{K}(\omega, s)\right)=(e-$ $\left.X_{s-}(\omega)\right) \mathbb{1}_{\left\{X_{s-}(\omega) \in\right] 0,1[\}}(\omega, s)$. 
Lemma 4.13. We set $E=[0,1]$. Let $(Y, U) \in \mathcal{L}^{2} \times \mathcal{G}^{2}(\mu)$ be a solution to the BSDE (4.31) and $X$ be the piecewise deterministic Markov process with local characteristics $(h, \lambda, P)$ satisfying (4.37). Assume that $Y_{t}=v\left(t, X_{t}\right)$ for some function $v:[0, T] \times \mathbb{R} \rightarrow \mathbb{R}$ such that its restriction to $[0, T] \times E$ is continuous. Then $(X, Y)$ satisfies Hypothesis 3.11 with corresponding function $v$.

Proof. By Remark 3.12, it follows that Hypothesis 3.11(iii) is verified. Taking into account Remark 3.13, it follows that $Y_{t}=v\left(t, X_{t}\right)$ has a finite number of jumps, so that $\sum_{s \leq T}\left|\Delta Y_{s}\right|<\infty$ a.s. On the other hand, by Remark 3.3, $Y$ is an $\left(\mathcal{F}_{t}\right)$-orthogonal process, so that Hypothesis 3.11(i) holds.

It remains to show that $v\left(t, X_{t}\right)$ satisfies condition (3.18). We have

$$
\begin{aligned}
\int_{] 0, \cdot] \times \mathbb{R}}\left|v\left(s, X_{s-}+x\right)-v\left(s, X_{s-}\right)\right| \mu^{X}(d s d x) & =\sum_{0<s \leq \cdot}\left|v\left(s, X_{s}\right)-v\left(s, X_{s-}\right)\right| \\
& =\sum_{s \leq \cdot}\left|\Delta Y_{s}\right|,
\end{aligned}
$$

by Remark 3.13. The process $Y$ takes values in the image of $[0, T] \times[0,1]$ with respect to $v$, which is a compact set. Therefore the jumps of $Y$ are bounded, and (4.52) belongs to $\mathcal{A}_{\text {loc }}^{+}$, see, for instance, the proof of Corollary at page 373 in [36].

Finally, we apply Theorem 3.14 to the present framework.

Corollary 4.14. Let $(Y, U) \in \mathcal{L}^{2} \times \mathcal{G}^{2}(\mu)$ be a solution to the BSDE (4.31), and $X$ the piecewise deterministic Markov process with local characteristics $(h, \lambda, P)$ satisfying (4.37). Assume that $Y_{t}=v\left(t, X_{t}\right)$ for some continuous function $v$. Assume in addition that there exists a function $\beta:\{0,1\} \rightarrow \mathbb{R}$, such that

$$
P(x, d e) \mathbb{1}_{\{x \in\{0,1\}\}}(s)=\delta_{\beta(x)}(d e) .
$$

Then the random field $U$ satisfies (3.19) with

$$
H_{s}(e):=\left(U_{s}(e)-\left(v(s, e)-v\left(s, X_{s-}\right)\right)\right) \mathbb{1}_{\left\{X_{s-} \in\right] 0,1[\}}(s)+U_{s}(e) \mathbb{1}_{\left\{X_{s-} \in\{0,1\}\right\}}(s) .
$$

If in addition $H_{s}(e) \in \mathcal{G}_{\mathrm{loc}}^{2}(\mu)$,

$$
U_{s}(e)=v(s, e)-v\left(s, X_{s-}\right) \quad d \mathbb{P} \lambda\left(X_{s-}\right) P\left(X_{s-}, d e\right) d s \text {-a.e. }
$$

and there exists a predictable process $\left(l_{s}\right)$ such that

$$
U_{s}(e)=l_{s} \mathbb{1}_{\left\{X_{s-} \in\{0,1\}\right\}}(s), \quad d \mathbb{P} \delta_{\beta\left(X_{s-}\right)}(d e) d p_{s}^{*} \text {-a.e. }
$$

Proof. We will apply Theorem 3.14. By Lemma 4.11, $\mu$ satisfies Hypothesis 2.14 and $(X, \mu)$ fulfills Hypothesis 2.9 with $\tilde{\gamma}(\omega, s, e)=\left(e-X_{s-}(\omega)\right) \mathbb{1}_{K}(\omega, s)$. Moreover, by Lemma 4.13, 
Hypothesis 3.11 holds for $(X, Y)$. We are then in condition to apply Theorem 3.14. Identity (3.19) holds with

$$
\begin{aligned}
H_{S}(e) & :=U_{s}(e)-\left[v\left(s, X_{s-}+\tilde{\gamma}(s, e)\right)-v\left(s, X_{s-}\right)\right] \\
& =U_{s}(e)-\left[v\left(s, X_{s-}+\left(e-X_{s-}\right) \mathbb{1}_{K}(s)\right)-v\left(s, X_{s-}\right)\right] \\
& =\left[U_{s}(e)-\left(v(s, e)-v\left(s, X_{s-}\right)\right)\right] \mathbb{1}_{K^{c}}(s)+U_{s}(e) \mathbb{1}_{K}(s) .
\end{aligned}
$$

At this point we recall that $v^{c}(d s d e)=\lambda\left(X_{s-}\right) P\left(X_{s-}, d e\right) d s$, see (4.47). Moreover, since $J=K$,

$$
v^{d}(d s d e)=v(d s d e) \mathbb{1}_{K}(s)=P\left(X_{s-}, d e\right) d p_{s}^{*}=\delta_{\beta\left(X_{s-}\right)}(d e) d p_{s}^{*} .
$$

Then, since by (4.42) we have $K=\left\{(\omega, s): X_{s-}(\omega) \in\{0,1\}\right\}$, (4.54) and (4.55) are direct consequences respectively, of (3.21) and (3.22).

\section{Appendix A: Technical results related to the hypothesis on the underlying process $X$}

The results below are related to Hypothesis 2.9 concerning $(X, \mu)$, with $X$ being a càdlàg process and $\mu$ an integer-valued random measure; they will be extensively used in Appendix B.

Remark A.1. (i) Given a predictable thin set $A$, there exists a sequence of predictable times $\left(R_{n}\right)_{n}$ with disjoint graphs, such that $A=\bigcup_{n}\left[\left[R_{n}\right]\right]$, up to an evanescent set, see Proposition 2.23, Chapter I, in [27].

(ii) Since $\left\{\Delta X^{p} \neq 0\right\}$ is a predictable thin set (see the comments after Definition 7.39 in [23]), by item (i) there exists a sequence of predictable times exhausting the jumps of $X^{p}$, up to an evanescent set.

Proposition A.2. Let $X$ be a càdlàg adapted process with decomposition $X=X^{i}+X^{p}$, where $X^{i}\left(\right.$ resp. $\left.X^{p}\right)$ is a càdlàg quasi-left continuous adapted process (resp. càdlàg predictable process). Then the two properties below hold.

(i) $\Delta X^{p} \mathbb{1}_{\left\{\Delta X^{i} \neq 0\right\}}=0$ and $\Delta X^{i} \mathbb{1}_{\left\{\Delta X^{p} \neq 0\right\}}=0$, up to an evanescent set.

(ii) $\{\Delta X \neq 0\}$ is the disjoint union (up to an evanescent set) of the random sets $\left\{\Delta X^{p} \neq 0\right\}$ and $\left\{\Delta X^{i} \neq 0\right\}$.

Proof. (i) By Remark A.1(ii), there exist a sequence of predictable times $\left(T_{n}^{p}\right)_{n}$ that exhausts the jumps of $X^{p}$, up to an evanescent set. Moreover, recalling Proposition 2.26, Chapter I, in [27], there exist a sequence of totally inaccessible times $\left(T_{n}^{i}\right)_{n}$ that exhausts the jumps of $X^{i}$. On the 
other hand, $\Delta X_{T_{n}^{i}}^{p}=0$ a.s. for every $n$, see Proposition 2.24, Chapter I, in [27] (resp. $\Delta X_{T_{n}^{p}}^{i}=0$ a.s. for every $n$, see Definition 2.25, Chapter I, in [27]), so that, up to an evanescent set,

$$
\Delta X^{i} \mathbb{1}_{\left\{\Delta X^{p} \neq 0\right\}}=\Delta X^{i} \mathbb{1}_{\bigcup_{n}\left[\left[T_{n}^{p}\right]\right]}=0, \quad \Delta X^{p^{p}} \mathbb{1}_{\left\{\Delta X^{i} \neq 0\right\}}=\Delta X^{p^{1}} \mathbb{1}_{\bigcup_{n}\left[\left[T_{n}^{i}\right]\right]}=0 .
$$

(ii) We have, again up to an evanescent set,

$$
\begin{aligned}
\{\Delta X \neq 0\} & =\left\{\left(\Delta X^{i}+\Delta X^{p}\right) \neq 0\right\} \\
& =\left(\left\{\left(\Delta X^{i}+\Delta X^{p}\right) \neq 0\right\} \cap\left\{\Delta X^{p}=0\right\}\right) \cup\left(\left\{\left(\Delta X^{i}+\Delta X^{p}\right) \neq 0\right\} \cap\left\{\Delta X^{p} \neq 0\right\}\right) \\
& =\left(\left\{\Delta X^{i} \neq 0\right\} \cap\left\{\Delta X^{p}=0\right\}\right) \cup\left\{\Delta X^{p} \neq 0\right\} \\
& =\left\{\Delta X^{i} \neq 0\right\} \cup\left\{\Delta X^{p} \neq 0\right\},
\end{aligned}
$$

where the third equality follows from the second statement in item (i) of the proposition. We observe that the intersection of $\left\{\Delta X^{i} \neq 0\right\}$ and $\left\{\Delta X^{i} \neq 0\right\}$ is evanescent because of item (i).

Proposition A.3. Let $X$ be a càdlàg adapted process with decomposition $X=X^{i}+X^{p}$, where $X^{i}\left(\right.$ resp. $\left.X^{p}\right)$ is a càdlàg quasi-left continuous adapted process (resp. càdlàg predictable process). Then

$$
\left\{(\omega, t): v^{X}(\omega,\{t\} \times \mathbb{R})>0\right\}=\left\{\Delta X^{p} \neq 0\right\}
$$

Proof. $\{\Delta X \neq 0\}$ is the support of the random measure $\mu^{X}$ (see, e.g., Proposition 1.16, Chapter II, in [27]). By Theorem 11.14 in [23], the predictable support of $\{\Delta X \neq 0\}$ is given by $\left\{(\omega, t): v^{X}(\{t\} \times \mathbb{R})>0\right\}$.

It remains to prove that the predictable support of $\{\Delta X \neq 0\}$ equals $\left\{\Delta X^{p} \neq 0\right\}$. By Proposition A.2(ii), $\{\Delta X \neq 0\}$ is the disjoint union (up to an evanescent set) of $\left\{\Delta X^{p} \neq 0\right\}$ and $\left\{\Delta X^{i} \neq 0\right\}$. Since $X^{i}$ is a càdlàg quasi-left continuous process, by Proposition 2.35, Chapter I, in [27], we know that the predictable support of $\left\{\Delta X^{i} \neq 0\right\}$ is evanescent. Then, by the definition of predictable support, see Definition 2.32, Chapter I, in [27], taking into account the additivity of the predictable projection operator, we have ${ }^{p}\left(\mathbb{1}_{\{\Delta X \neq 0\}}\right)=\mathbb{1}_{\left\{\Delta X^{p} \neq 0\right\}}$, and this concludes the proof.

Proposition A.4. Let $X$ be a càdlàg adapted process with decomposition $X=X^{i}+X^{p}$, where $X^{i}$ (resp. $\left.X^{p}\right)$ is a càdlàg quasi-left-continuous adapted process (resp. càdlàg predictable process). Let $\left(S_{n}\right)_{n}$ be a sequence of predictable times exhausting the jumps of $X^{p}$. Then

$$
v^{X}\left(\left\{S_{n}\right\}, d x\right)=\mu^{X}\left(\left\{S_{n}\right\}, d x\right) \quad \text { for any } n \text {, a.s. }
$$

Proof. Let us fix $n \in \mathbb{N}$. We need to show the existence of a $\mathbb{P}$-null set $\mathcal{N}$ such that, for every $\omega \notin \mathcal{N}$, we have

$$
\int_{\mathbb{R}} \mathbb{1}_{E_{m}}(x) v^{X}\left(\left\{S_{n}\right\}, d x\right)=\int_{\mathbb{R}} \mathbb{1}_{E}(x) \mu^{X}\left(\left\{S_{n}\right\}, d x\right)
$$


for every real Borel set $E$. Let $\left(E_{m}\right)_{m}$ be a sequence of measurable subsets of $\mathbb{R}$ which is a $\pi$ class generating $\mathcal{B}(\mathbb{R})$. Since $X^{i}$ is a càdlàg quasi-left-continuous adapted process and $S_{n}$ is a predictable time, then $\Delta X_{S_{n}}^{i}=0$ a.s., see again Definition 2.25, Chapter I, in [27]. This implies that $\Delta X_{S_{n}}=\Delta X_{S_{n}}^{p}$ a.s. by Hypothesis 2.9-2. Consequently, for every $m$ we have

$$
\mathbb{1}_{E_{m}}\left(\Delta X_{S_{n}}^{p}\right)=\mathbb{1}_{E_{m}}\left(\Delta X_{S_{n}}\right)=\int_{\mathbb{R}} \mathbb{1}_{E_{m}}(x) \mu^{X}\left(\left\{S_{n}\right\}, d x\right) \quad \text { a.s. }
$$

On the other hand, by point (b) of Proposition 1.17, Chapter II, in [27] and (A.3) we have

$$
\begin{aligned}
\int_{\mathbb{R}} \mathbb{1}_{E_{m}}(x) v^{X}\left(\left\{S_{n}\right\}, d x\right) & =\mathbb{E}\left[\int_{\mathbb{R}} \mathbb{1}_{E_{m}}(x) \mu^{X}\left(\left\{S_{n}\right\}, d x\right) \mid \mathcal{F}_{S_{n}-}\right] \\
& =\mathbb{E}\left[\mathbb{1}_{E_{m}}\left(\Delta X_{S_{n}}^{p}\right) \mid \mathcal{F}_{S_{n}-}\right] \\
& =\mathbb{1}_{E_{m}}\left(\Delta X_{S_{n}}^{p}\right) \quad \text { a.s. }
\end{aligned}
$$

By (A.3), there exists a null set $\mathcal{N}_{m}$ such that

$$
\int_{\mathbb{R}} \mathbb{1}_{E_{m}}(x) v^{X}\left(\left\{S_{n}\right\}, d x\right)=\int_{\mathbb{R}} \mathbb{1}_{E_{m}}(x) \mu^{X}\left(\left\{S_{n}\right\}, d x\right) \quad \text { for every } \omega \notin \mathcal{N}_{m} .
$$

Define $\mathcal{N}=\bigcup_{m} \mathcal{N}_{m}$, then

$$
\int_{\mathbb{R}} \mathbb{1}_{E_{m}}(x) v^{X}\left(\left\{S_{n}\right\}, d x\right)=\int_{\mathbb{R}} \mathbb{1}_{E_{m}}(x) \mu^{X}\left(\left\{S_{n}\right\}, d x\right) \quad \text { for every } m \text { and } \omega \notin \mathcal{N} .
$$

Then (A.2) follows by a monotone class argument, see Theorem 1.1, Chapter 1, [29].

Proposition A.5. Let $Y$ be a càdlàg adapted process such that $(Y, \mu)$ satisfies Hypothesis 2.9-1. Then, there exists a null set $\mathcal{N}$ such that, for every Borel function $\varphi:[0, T] \times \mathbb{R} \rightarrow \mathbb{R}_{+}$satisfying $\varphi(s, 0)=0$ for every $s \in[0, T]$, we have

$$
\sum_{0<s \leq T} \varphi\left(s, \Delta Y_{s}(\omega)\right)=\int_{] 0, T] \times \mathbb{R}} \varphi(s, \tilde{\gamma}(\omega, s, e)) \mu(\omega, d s d e), \quad \omega \notin \mathcal{N} .
$$

Proof. Taking into account that $\{\Delta Y \neq 0\} \subset D$ and the fact that $\varphi(s, 0)=0$, it will be enough to prove that

$$
\begin{aligned}
& \sum_{0<s \leq T} \varphi\left(s, \Delta Y_{s}(\omega)\right) \mathbb{1}_{D}(\omega, s) \\
& =\int_{] 0, T] \times \mathbb{R}} \varphi(s, \tilde{\gamma}(\omega, s, e)) \mu(\omega, d s d e), \quad \omega \notin \mathcal{N},
\end{aligned}
$$

for every Borel function $\varphi:[0, T] \times \mathbb{R} \rightarrow \mathbb{R}_{+}$. 
Let $\left(I_{m}\right)_{m}$ be a sequence of subsets of $[0, T] \times \mathbb{R}$, which is a $\pi$-system generating $\mathcal{B}([0, T]) \otimes$ $\mathcal{B}(\mathbb{R})$. Setting $\varphi_{m}(s, x)=\mathbb{1}_{I_{m}}(s, x)$, for every $m$, we will show that

$$
\sum_{0<s \leq T} \varphi_{m}\left(s, \Delta Y_{s}\right) \mathbb{1}_{D}(\cdot, s)=\int_{] 0, T] \times \mathbb{R}} \varphi_{m}(s, \tilde{\gamma}(\cdot, s, e)) \mu(\cdot, d s d e), \quad \text { a.s. }
$$

Let $n \in \mathbb{N}$ be fixed. In order to establish (A.6), it is enough to prove

$$
\begin{aligned}
& \sum_{0<s \leq T} \varphi_{m}\left(s, \Delta Y_{s}\right) \mathbb{1}_{\tilde{\Omega}_{n}}(\cdot, s) \mathbb{1}_{D}(\cdot, s) \\
& \quad=\int_{] 0, T] \times \mathbb{R}} \varphi_{m}(s, \tilde{\gamma}(\cdot, s, e)) \mathbb{1}_{\tilde{\Omega}_{n}}(\cdot, s) \mu(\cdot, d s d e), \quad \text { a.s., }
\end{aligned}
$$

where $\tilde{\Omega}_{n}$ are the sets introduced in Definition 2.3. Let us consider a bounded, $\mathcal{F}$-measurable function $\phi: \Omega \rightarrow \mathbb{R}_{+}$. Identity (A.7) holds if we show that the expectations of both sides against $\phi$ are equal. Using Hypothesis 2.9-1., we write

$$
\begin{aligned}
\mathbb{E} & {\left[\phi \int_{] 0, T] \times \mathbb{R}} \varphi_{m}(s, \tilde{\gamma}(\cdot, s, e)) \mathbb{1}_{\tilde{\Omega}_{n}}(\cdot, s) \mu(\cdot, d s d e)\right] } \\
& =\int_{\Omega \times] 0, T] \times \mathbb{R}} d M_{\mu}^{\mathbb{P}}(\omega, s, e) \phi(\omega) \varphi_{m}(s, \tilde{\gamma}(\omega, s, e)) \mathbb{1}_{\tilde{\Omega}_{n}}(\omega, s) \\
& =\int_{\Omega \times] 0, T]} d M_{\mu}^{\mathbb{P}}(\omega, s, y) \phi(\omega) \varphi_{m}\left(s, \Delta Y_{s}(\omega)\right) \mathbb{1}_{\tilde{\Omega}_{n}}(\omega, s) \\
& =\mathbb{E}\left[\phi \int_{] 0, T] \times \mathbb{R}} \varphi_{m}\left(s, \Delta Y_{s}(\cdot)\right) \mathbb{1}_{\tilde{\Omega}_{n}}(\cdot, s) \mu(\cdot, d s d y)\right] \\
& =\mathbb{E}\left[\phi \int_{] 0, T] \times \mathbb{R}} \sum_{s>0} \varphi_{m}\left(s, \Delta Y_{s}(\cdot)\right) \mathbb{1}_{\tilde{\Omega}_{n}}(\cdot, s) \mathbb{1}_{D}(\cdot, s) \delta_{\left(s, \beta_{s}(\cdot)\right)}(d t d x)\right] \\
& =\mathbb{E}\left[\phi \sum_{0<s \leq T} \mathbb{1}_{D}(\cdot, s) \varphi_{m}\left(s, \Delta Y_{s}(\cdot)\right) \mathbb{1}_{\tilde{\Omega}_{n}}(\cdot, s)\right],
\end{aligned}
$$

where we have used the form of $\mu$ given in Proposition 1.14, Chapter II, in [27], that is,

$$
\mu(d t d y)=\sum_{s \geq 0} \mathbb{1}_{D}(s, \omega) \delta_{\left(s, \beta_{s}(\omega)\right)}(d t d y) .
$$

Therefore, there exists a $\mathbb{P}$-null set $\mathcal{N}_{m}$ such that

$$
\begin{aligned}
& \sum_{0<s \leq T} \varphi_{m}\left(s, \Delta Y_{s}(\omega)\right) \mathbb{1}_{D}(\omega, s) \mathbb{1}_{\tilde{\Omega}_{n}}(\omega, s) \\
& \quad=\int_{10, T] \times \mathbb{R}} \varphi_{m}(s, \tilde{\gamma}(\omega, s, e)) \mathbb{1}_{\tilde{\Omega}_{n}}(\omega, s) \mu(\omega, d s d e), \quad \omega \notin \mathcal{N}_{m} .
\end{aligned}
$$


Define $\mathcal{N}=\cup_{m} \mathcal{N}_{m}$, then for $\varphi=\varphi_{m}$ for every $m$ we have

$$
\begin{aligned}
& \sum_{0<s \leq T} \varphi\left(s, \Delta Y_{s}(\omega)\right) \mathbb{1}_{D}(\omega, s) \mathbb{1}_{\tilde{\Omega}_{n}}(\omega, s) \\
& \quad=\int_{] 0, T] \times \mathbb{R}} \varphi(s, \tilde{\gamma}(\omega, s, e)) \mathbb{1}_{\tilde{\Omega}_{n}}(\omega, s) \mu(\omega, d s d e), \quad \omega \notin \mathcal{N} .
\end{aligned}
$$

By a monotone class argument (see Theorem 2.3 in [26]) the identity holds for every measurable bounded function $\varphi:[0, T] \times \mathbb{R} \rightarrow \mathbb{R}$, and therefore, using monotone convergence theorem, for every positive measurable function $\varphi$ on $[0, T] \times \mathbb{R}$ as well.

\section{Appendix B: Proofs of the technical results stated in Section 2.2}

\section{B.1. Proof of Proposition 2.12}

Let $\varphi:[0, T] \times \mathbb{R} \rightarrow \mathbb{R}_{+}$. Taking into account Proposition A.2(i) and the fact that $\varphi(s, 0)=0$, we have, for almost all $\omega$,

$$
\begin{aligned}
\sum_{0<s \leq T} \varphi\left(s, \Delta X_{s}(\omega)\right)= & \sum_{0<s \leq T} \varphi\left(s, \Delta X_{s}^{i}(\omega)+\Delta X_{s}^{p}(\omega)\right) \mathbb{1}_{\left\{\Delta X^{p}=0\right\}}(\omega, s) \\
& +\sum_{0<s \leq T} \varphi\left(s, \Delta X_{s}^{i}(\omega)+\Delta X_{s}^{p}(\omega)\right) \mathbb{1}_{\left\{\Delta X^{p} \neq 0\right\}}(\omega, s) \\
= & \sum_{0<s \leq T} \varphi\left(s, \Delta X_{s}^{i}(\omega)\right) \mathbb{1}_{\left\{\Delta X^{p}=0\right\}}(\omega, s) \\
& +\sum_{0<s \leq T} \varphi\left(s, \Delta X_{s}^{p}(\omega)\right) \mathbb{1}_{\left\{\Delta X^{p} \neq 0\right\}}(\omega, s) \\
= & \sum_{0<s \leq T} \varphi\left(s, \Delta X_{s}^{i}(\omega)\right)+\sum_{0<s \leq T} \varphi\left(s, \Delta X_{s}^{p}(\omega)\right) .
\end{aligned}
$$

By Proposition A.5 applied to $Y=X^{i}$, there exists a null set $\mathcal{N}$ such that, for every $\omega \notin \mathcal{N}$, previous expression gives

$$
\int_{] 0, T] \times \mathbb{R}} \varphi(s, x) \mu^{X}(\omega, d s d x)=\int_{] 0, T] \times \mathbb{R}} \varphi(s, \tilde{\gamma}(\omega, s, e)) \mu(\omega, d s d e)+\sum_{0<s \leq T} \varphi\left(s, \Delta X_{s}^{p}(\omega)\right) .
$$

The second part of the statement holds decomposing $\varphi=\varphi^{+}-\varphi^{-}$.

\section{B.2. Proof of Proposition 2.17}

Clearly the result holds if we show that $\varphi$ verifies (2.19) under one of the two following assumptions: 
(i) $|\varphi| \star \mu^{X} \in \mathcal{A}_{\text {loc }}^{+}$,

(ii) $|\varphi|^{2} \star \mu^{X} \in \mathcal{A}_{\text {loc }}^{+}$.

By localization arguments, it is enough to show it when $|\varphi| \star \mu^{X} \in \mathcal{A}^{+},|\varphi|^{2} \star \mu^{X} \in \mathcal{A}^{+}$. Below we will consider the first case, the second case will follow from the first one by approaching in $\mathcal{L}^{2}\left(\mu^{X}\right)$ the function $\varphi$ with $\varphi_{\varepsilon}(s, x):=\varphi(s, x) \mathbb{1}_{\varepsilon<|x| \leq 1 / \varepsilon} \mathbb{1}_{s \in[0, T]}$. Indeed, $\varphi_{\varepsilon}(s, x) \star \mu \in \mathcal{A}^{+}$, by Cauchy-Schwarz inequality, taking into account the fact that $\mu^{X}$, restricted to $\varepsilon \leq|x| \leq 1 / \varepsilon$, is finite, since $\mu^{X}$ is $\sigma$-finite on $[0, \infty) \times \mathbb{R}$.

Let us define

$$
\begin{aligned}
& M_{t}:=\int_{] 0, t] \times \mathbb{R}} \varphi(\cdot, s, x)\left(\mu^{X}-v^{X}\right)(d s d x), \\
& N_{t}:=\int_{] 0, t] \times \mathbb{R}} \varphi(\cdot, s, \tilde{\gamma}(\cdot, s, e))(\mu-v)(d s d e) .
\end{aligned}
$$

Notice that the processes $M$ and $N$ are purely discontinuous local martingales, see e.g. Definition 1.27 , point b), Chapter II, in [27]. We have to prove that $M$ and $N$ are indistinguishable. To this end, by Corollary 4.19, Chapter I, in [27], it is enough to prove that $\Delta M=\Delta N$, up to an evanescent set. Observe that

$$
\begin{aligned}
\Delta M_{s}= & \int_{\mathbb{R}} \varphi(\cdot, s, x)\left(\mu^{X}-v^{X}\right)(\{s\}, d x) \\
= & \int_{\mathbb{R}} \varphi(\cdot, s, x)\left(1-\mathbb{1}_{J}(\cdot, s)\right)\left(\mu^{X}-v^{X}\right)(\{s\}, d x) \\
& +\int_{\mathbb{R}} \varphi(\cdot, s, x) \mathbb{1}_{J}(\cdot, s)\left(\mu^{X}-v^{X}\right)(\{s\}, d x),
\end{aligned}
$$

and

$$
\begin{aligned}
\Delta N_{s}= & \int_{\mathbb{R}} \varphi(\cdot, s, \tilde{\gamma}(\cdot, s, e))(\mu-v)(\{s\}, d e) \\
= & \int_{\mathbb{R}} \varphi(\cdot, s, \tilde{\gamma}(\cdot, s, e)) \mathbb{1}_{J}(\cdot, s)(\mu-v)(\{s\}, d e) \\
& +\int_{\mathbb{R}} \varphi(\cdot, s, \tilde{\gamma}(\cdot, s, e))\left(1-\mathbb{1}_{J}(\cdot, s)\right)(\mu-v)(\{s\}, d e) .
\end{aligned}
$$

By definition of $J$, for every $\omega$ and every $s$ we have

$$
v(\omega,\{s\}, d e)\left(1-\mathbb{1}_{J}(\omega, s)\right)=0 .
$$

Moreover, since $J$ is a predictable thin set (see Remark 2.1), there exists a sequence of predictable times $\left(R_{n}\right)_{n}$ with disjoint graphs, such that $J=\bigcup_{n}\left[\left[R_{n}\right]\right]$, see Remark A.1(i). We recall that Hypothesis 2.14(i) implies that $J=K$ up to an evanescent set, see Remark 2.15. By this fact, 
and taking into account Hypothesis 2.14(ii), there exists a null set $\mathcal{N}$, such that, for every $n \in \mathbb{N}$, $\omega \notin \mathcal{N}$,

$$
\mu\left(\omega,\left\{R_{n}(\omega)\right\}, d e\right) \mathbb{1}_{J}(\omega, s)=v\left(\omega,\left\{R_{n}(\omega)\right\}, d e\right) \mathbb{1}_{J}(\omega, s) .
$$

By additivity, it follows that for every $\omega \notin \mathcal{N}$, for every $s \in[0, T]$,

$$
\mu(\omega,\{s\}, d e) \mathbb{1}_{J}(\omega, s)=v(\omega,\{s\}, d e) \mathbb{1}_{J}(\omega, s) .
$$

On the other hand, $\left\{\Delta X^{p} \neq 0\right\} \subset J$ by Hypothesis 2.9-2. Recalling that $\left\{\Delta X^{p} \neq 0\right\}=\{(\omega, s)$ : $\left.v^{X}(\{s\} \times \mathbb{R})>0\right\}($ see Proposition A.3), for almost every $\omega$, for every $s \in[0, T]$, we have

$$
v^{X}(\omega,\{s\}, d x) \mathbb{1}_{J}(\omega, s)=v^{X}(\omega,\{s\}, d x) \mathbb{1}_{\left\{\Delta X^{p} \neq 0\right\}}(\omega, s),
$$

so that

$$
v^{X}(\omega,\{s\}, d x)\left(1-\mathbb{1}_{J}(\omega, s)\right)=v^{X}(\omega,\{s\}, d x)\left(1-\mathbb{1}_{\left\{\Delta X^{p} \neq 0\right\}}(\omega, s)\right)=0 .
$$

Now notice that there always exists a sequence of predictable times exhausting the jumps of $X^{p}$, up to an evanescent set, see Remark A.1(ii). By means of Proposition A.4, we can prove, similarly as we did in order to establish (B.5), that for every $\omega \notin \mathcal{N}(\mathcal{N}$ possibly enlarged), for every $s \in[0, T]$,

$$
\mu^{X}(\omega,\{s\}, d x) \mathbb{1}_{\left\{\Delta X^{p} \neq 0\right\}}(\omega, s)=v^{X}(\omega,\{s\}, d x) \mathbb{1}_{\left\{\Delta X^{p} \neq 0\right\}}(\omega, s) .
$$

Finally, we notice that

$$
\mu^{X}(\omega,\{s\}, d x) \mathbb{1}_{J}(\omega, s)=\mu^{X}(\omega,\{s\}, d x) \mathbb{1}_{J \cap\{\Delta X \neq 0\}}(\omega, s) .
$$

Taking into account that $X^{i}$ is a càdlàg quasi-left-continuous process, by Definition 2.25, Chapter I, in [27] we have $\Delta X_{R_{n}}^{i}=0$ for every $n$, so that

$$
\begin{aligned}
J \cap\{\Delta X \neq 0\} & =\left(\bigcup_{n}\left[\left[R_{n}\right]\right] \cap\left\{\Delta X^{i} \neq 0\right\}\right) \cup\left(\bigcup_{n}\left[\left[R_{n}\right]\right] \cap\left\{\Delta X^{p} \neq 0\right\}\right) \\
& =\bigcup_{n}\left[\left[R_{n}\right]\right] \cap\left\{\Delta X^{p} \neq 0\right\}=\left\{\Delta X^{p} \neq 0\right\} .
\end{aligned}
$$

This, together with (B.9), implies that, for every $\omega \notin \mathcal{N}$ and for every $s \in[0, T]$,

$$
\begin{aligned}
\mu^{X}(\omega,\{s\}, d x) \mathbb{1}_{J}(\omega, s) & =\mu^{X}(\omega,\{s\}, d x) \mathbb{1}_{J \cap\{\Delta X \neq 0\}}(\omega, s) \\
& =\mu^{X}(\omega,\{s\}, d x) \mathbb{1}_{\left\{\Delta X^{p} \neq 0\right\}}(\omega, s) .
\end{aligned}
$$

Collecting (B.6), (B.8) and (B.10) we conclude that for every $\omega \notin \mathcal{N}$, for every $s \in[0, T]$,

$$
\mu^{X}(\omega,\{s\}, d x) \mathbb{1}_{J}(\omega, s)=v^{X}(\omega,\{s\}, d x) \mathbb{1}_{J}(\omega, s) .
$$


Therefore, for every $\omega \notin \mathcal{N}$, for every $s \in[0, T]$, taking into account (B.4), (B.5), (B.7), (B.11), expressions (B.2) and (B.3) become

$$
\begin{aligned}
\Delta M_{s} & =\int_{\mathbb{R}} \varphi(\cdot, s, x)\left(1-\mathbb{1}_{J}(\cdot, s)\right) \mu^{X}(\{s\}, d x), \\
\Delta N_{s} & =\int_{\mathbb{R}} \varphi(\cdot, s, \tilde{\gamma}(\cdot, s, e))\left(1-\mathbb{1}_{J}(\cdot, s)\right) \mu(\{s\}, d e) .
\end{aligned}
$$

Now let us prove that, for every $s \in[0, T], \Delta M_{s}(\omega)=\Delta N_{s}(\omega)$ for every $\omega \notin \mathcal{N}$, namely up to an evanescent set. Set

$$
\varphi_{s}(\omega, t, x):=\varphi(\omega, t, x)\left(1-\mathbb{1}_{J}(\omega, t)\right) \mathbb{1}_{\{s\}}(t)
$$

then $\Delta M_{S}$ and $\Delta N_{s}$ can be rewritten as

$$
\begin{aligned}
& \Delta M_{S}(\omega)=\int_{[0, T] \times \mathbb{R}} \varphi_{s}(\omega, t, x) \mu^{X}(\omega, d t d x), \\
& \Delta N_{S}(\omega)=\int_{[0, T] \times \mathbb{R}} \varphi_{s}(\omega, t, \tilde{\gamma}(\omega, t, e)) \mu(\omega, d t d e) .
\end{aligned}
$$

Then, Proposition 2.12 and Remark 2.13 applied to the process $\varphi_{s}$ implies that (possibly enlarging the null set $\mathcal{N}$ ),

$$
\int_{[0, T] \times \mathbb{R}} \varphi_{s}(\omega, t, x) \mu^{X}(\omega, d t d x)=\int_{] 0, T] \times \mathbb{R}} \varphi_{s}(t, \tilde{\gamma}(\omega, t, e)) \mu(\omega, d t d e)+V^{\varphi_{s}}(\omega)
$$

for every $\omega \notin \mathcal{N}$, or, equivalently, that

$$
\int_{\mathbb{R}} \varphi(\omega, s, x) \mu^{X}(\omega,\{s\}, d x)=\int_{\mathbb{R}} \varphi(\omega, s, \tilde{\gamma}(\omega, s, e)) \mu(\omega,\{s\}, d e)+V^{\varphi_{s}}(\omega),
$$

for every $\omega \notin \mathcal{N}$, where

$$
V^{\varphi_{s}}(\omega)=\sum_{t \leq T} \varphi_{s}\left(\omega, t, \Delta X_{t}^{p}(\omega)\right)=\varphi\left(\omega, s, \Delta X_{s}^{p}(\omega)\right) \mathbb{1}_{J^{c} \cap\left\{\Delta X^{p} \neq 0\right\}}(\omega, s) .
$$

Recalling that $\left\{\Delta X^{p} \neq 0\right\} \subset J$ by Hypothesis 2.9-2., it straightly follows from (B.14) that $V^{\varphi_{s}}(\omega)$ is zero. In particular, up to an evanescent set, we have

$$
\int_{\mathbb{R}} \varphi(\omega, s, x) \mu^{X}(\omega,\{s\}, d x)=\int_{\mathbb{R}} \varphi(s, \tilde{\gamma}(\omega, s, e)) \mu(\omega,\{s\}, d e),
$$

in other words $\Delta M=\Delta N$ up to an evanescent set, and this concludes the proof. 


\section{B.3. Proof of Lemma 2.19}

Since $N$ is continuous, it straight follows from (2.25) that

$$
\Delta X_{s}^{p}=b\left(s, X_{s-}\right) \Delta B_{s} .
$$

We remark that $X^{i}$ in (2.24) has the same expression as $N$ defined in (B.1) where the integrand $\varphi(\omega, s, \tilde{\gamma}(\omega, s, e))$ is replaced by $\gamma\left(s, X_{s-}(\omega), e\right)$. We recall that Hypothesis 2.14(i) implies that $J=K$ up to an evanescent set, see Remark 2.15. Similarly as for (B.13), we get

$$
\Delta X_{s}^{i}=\int_{\mathbb{R}} \gamma\left(s, X_{s-}, e\right)\left(1-\mathbb{1}_{K}(s)\right) \mu(\{s\}, d e) .
$$

Since by Hypothesis 2.14, up to an evanescent set, $D \backslash K=\bigcup_{n}\left[\left[T_{n}^{i}\right]\right],\left(T_{n}^{i}\right)_{n}$ being a sequence of totally inaccessible times with disjoint graphs, recalling (A.8), (B.16) can be rewritten as

$$
\Delta X_{s}^{i}(\omega)=\gamma\left(s, X_{s-}(\omega), \beta_{s}(\omega)\right) \mathbb{1}_{\bigcup_{n}\left[\left[T_{n}^{i}\right]\right]}(\omega, s) .
$$

We can easily show that $X^{p}$ and $X^{i}$ are respectively a càdlàg predictable process and a càdlàg quasi-left-continuous adapted process. The fact that $X^{p}$ is predictable straight follow from (2.25). Concerning $X^{i}$, let $S$ be a predictable time; it is enough to prove that $\Delta X_{S}^{i} \mathbb{1}_{\{S<\infty\}}=0$ a.s., see Definition 2.25, Chapter I, in [27]. Identity (B.17) gives

$$
\Delta X_{S}^{i}(\omega) \mathbb{1}_{\{S<\infty\}}=\gamma\left(S, X_{S-}(\omega), \beta_{S}(\omega)\right) \mathbb{1}_{\bigcup_{n}\left[\left[T_{n}^{i}\right]\right]}(\omega, S(\omega)) \mathbb{1}_{\{S<\infty\}}
$$

Since the graphs of the totally inaccessible times $T_{n}^{i}$ are disjoint, $\mathbb{1}_{\cup_{n}\left[\left[T_{n}^{i}\right]\right]}(\omega, S(\omega)) \mathbb{1}_{\{S<\infty\}}=$ $\sum_{n} \mathbb{1}_{\left[\left[T_{n}^{i}\right]\right]}(\omega, S(\omega)) \mathbb{1}_{\{S<\infty\}}$, and the conclusion follows by Remark 2.2(iii), taking into account that $S$ is a predictable time.

The process $X^{p}$ in (2.25) satisfies Hypothesis 2.9-2. Indeed, by (B.15) we have

$$
\left\{\Delta X^{p} \neq 0\right\} \subset\{\Delta B \neq 0\} \subset J .
$$

Finally, we show that the process $X^{i}$ in (2.24) fulfills Hypothesis 2.9-1. with $\tilde{\gamma}(\omega, s, e)=$ $\gamma\left(s, X_{s-}(\omega), e\right)\left(1-\mathbb{1}_{K}(\omega, s)\right)$. First, the fact that $\left\{\Delta X^{i} \neq 0\right\} \subset D$ directly follows from (B.16). To prove $\Delta X_{s}^{i}(\omega)=\tilde{\gamma}(\omega, s, \cdot), d M_{\mu}^{\mathbb{P}}(\omega, s)$-a.e. it is enough to show that

$$
\mathbb{E}\left[\int_{] 0, T] \times \mathbb{R}} \mu(\omega, d s d e)\left|\tilde{\gamma}(\omega, s, e)-\Delta X_{s}^{i}(\omega)\right| \mathbb{1}_{\tilde{\Omega}_{n}}(\omega, s)\right]=0 .
$$

To establish this, we see that by the structure of $\mu$ it follows that, for every $n \in \mathbb{N}$,

$$
\begin{aligned}
& \mathbb{E}\left[\int_{] 0, T] \times \mathbb{R}} \mu(\omega, d s d e)\left|\tilde{\gamma}(\omega, s, e)-\Delta X_{s}^{i}(\omega)\right| \mathbb{1}_{\tilde{\Omega}_{n}}(\omega, s)\right] \\
& \quad \leq \sum_{s \in] 0, T]} \mathbb{E}\left[\mathbb{1}_{D}(\cdot, s)\left|\tilde{\gamma}\left(\cdot, s, \beta_{s}(\cdot)\right)-\Delta X_{s}^{i}(\cdot)\right|\right],
\end{aligned}
$$

which vanishes taking into account (B.17). 


\section{Acknowledgements}

The authors wish to thank the Referees for their valuable comments and criticisms, which have motivated us to strongly improve the first submitted version of the paper. The first named author benefited from the support of the Italian MIUR-PRIN 2010-11 "Evolution differential problems: deterministic and stochastic approaches and their interactions". The second named author benefited partially from the support of the "FMJH Program Gaspard Monge in optimization and operation research" (Project 2014-1607H).

\section{References}

[1] Bandini, E. Optimal control of Piecewise-Deterministic Markov Processes: A BSDE representation of the value function. In ESAIM: Control, Optimisation and Calculus of Variations. To appear. DOI: $10.1051 / \mathrm{cocv} / 2017009$.

[2] Bandini, E. (2015). Existence and uniqueness for BSDEs driven by a general random measure, possibly non quasi-left-continuous. Electron. Commun. Probab. 20 no. 71, 13. MR3407215

[3] Bandini, E. and Confortola, F. (2017). Optimal control of semi-Markov processes with a backward stochastic differential equations approach. Math. Control Signals Systems 29 Art. 1, 35. MR3584592

[4] Bandini, E. and Fuhrman, M. (2017). Constrained BSDEs representation of the value function in optimal control of pure jump Markov processes. Stochastic Process. Appl. 127 1441-1474. MR3630231

[5] Bandini, E. and Russo, F. (2017). Weak Dirichlet processes with jumps. Stochastic Process. Appl. DOI:10.1016/j.spa.2017.04.001.

[6] Barles, G., Buckdahn, R. and Pardoux, E. (1997). Backward stochastic differential equations and integral-partial differential equations. Stochastic Rep. 60 57-83.

[7] Becherer, D. (2006). Bounded solutions to backward SDE's with jumps for utility optimization and indifference hedging. Ann. Appl. Probab. 16 2027-2054. MR2288712

[8] Buckdahn, R. (1993). Backward stochastic differential equations driven by a martingale. Unpublished manuscript.

[9] Buckdahn, R. and Pardoux, E. (1994). BSDE's with jumps and associated integral-stochastic differential equations. Preprint.

[10] Carbone, R., Ferrario, B. and Santacroce, M. (2008). Backward stochastic differential equations driven by càdlàg martingales. Theory Probab. Appl. 52 304-314.

[11] Ceci, C., Cretarola, A. and Russo, F. (2014). BSDEs under partial information and financial applications. Stochastic Process. Appl. 124 2628-2653.

[12] Cohen, S. and Elliott, R. (2012). Existence, uniqueness and comparisons for BSDEs in general spaces. Ann. Probab. 40 2264-2297.

[13] Confortola, F. and Fuhrman, M. (2014). Backward stochastic differential equations associated to Markov jump processes and applications. Stochastic Processes and Their Applications 124 289-316.

[14] Confortola, F., Fuhrman, M. and Jacod, J. (2016). Backward stochastic differential equation driven by a marked point process: An elementary approach with an application to optimal control. Ann. Appl. Probab. 26 1743-1773.

[15] Davis, M.H.A. (1993). Markov Models and Optimization. Monographs on Statistics and Applied Probability 49. London: Chapman \& Hall. MR1283589

[16] Delong, L. (2013). Backward Stochastic Differential Equations with Jumps and Their Actuarial and Financial Applications. Berlin: Springer. 
[17] El Karoui, N. and Huang, S.-J. (1997). A general result of existence and uniqueness of backward stochastic differential equations. In Backward Stochastic Differential Equations (Paris, 1995-1996). Pitman Res. Notes Math. Ser. 364 27-36. Longman, Harlow. MR1752673

[18] Flandoli, F., Issoglio, E. and Russo, F. (2017). Multidimensional stochastic differential equations with distributional drift. Trans. Amer. Math. Soc. 369 1665-1688.

[19] Flandoli, F., Russo, F. and Wolf, J. (2003). Some SDEs with distributional drift. I. General calculus. Osaka J. Math. 40 493-542. MR1988703

[20] Fuhrman, M. and Tessitore, G. (2003). Generalized directional gradients, backward stochastic differential equations and mild solutions of semilinear parabolic equations. Appl. Math. Optim. 108263 298.

[21] Gozzi, F. and Russo, F. (2006). Verification theorems for stochastic optimal control problems via a time dependent Fukushima-Dirichlet decomposition. Stochastic Process. Appl. 116 1530-1562. MR2269215

[22] Gozzi, F. and Russo, F. (2006). Weak Dirichlet processes with a stochastic control perspective. Stochastic Process. Appl. 116 1563-1583. MR2269216

[23] He, S., Wang, J. and Yan, J. (1992). Semimartingale Theory and Stochastic Calculus. New York: Science Press Bejiing.

[24] Jacod, J. (1976). Un théorème de représentation pour les martingales discontinues. Z. Wahrsch. Verw. Gebiete 34 225-244. MR0418222

[25] Jacod, J. (1979). Calcul Stochastique et Problèmes de Martingales. Lecture Notes in Math. 714. Berlin: Springer. MR0542115

[26] Jacod, J. and Protter, P.E. (2003). Probability Essentials. Berlin: Springer.

[27] Jacod, J. and Shiryaev, A.N. (2003). Limit Theorems for Stochastic Processes, 2nd ed. Grundlehren der Mathematischen Wissenschaften [Fundamental Principles of Mathematical Sciences] 288. Berlin: Springer.

[28] Jeanblanc, M., Mania, M., Santacroce, M. and Schweizer, M. (2012). Mean-variance hedging via stochastic control and BSDEs for general semimartingales. Ann. Appl. Probab. 22 2388-2428. MR3024972

[29] Kallenberg, O. (1997). Foundations of Modern Probability. Probability and Its Applications (New York). New York: Springer.

[30] Laachir, I. and Russo, F. (2016). BSDEs càdlàg martingale problems, and orthogonalization under basis risk. SIAM J. Financial Math. 7 308-356.

[31] Pardoux, É. and Peng, S. (1990). Adapted solution of a backward stochastic differential equation. Systems Control Lett. 14 55-61.

[32] Pardoux, É. and Peng, S. (1992). Backward stochastic differential equations and quasilinear parabolic partial differential equations. Lecture Notes in CIS 176 200-217.

[33] Pardoux, E. and Răşcanu, A. (2014). Stochastic Differential Equations, Backward SDEs, Partial Differential Equations. Stochastic Modelling and Applied Probability 69. Cham: Springer. MR3308895

[34] Peng, S. (1991). Probabilistic interpretation for systems of quasilinear parabolic partial differential equations. Stochastics 37 61-74.

[35] Peng, S. (1992). A generalized dynamic programming principle and Hamilton-Jacobi-Bellman Equation. Stochastics 38 119-134.

[36] Protter, P.E. (2005). Stochastic Integration and Differential Equations. 2nd ed. Stochastic Modelling and Applied Probability 21. Berlin: Springer. MR2273672

[37] Russo, F. and Trutnau, G. (2007). Some parabolic PDEs whose drift is an irregular random noise in space. Ann. Probab. 35 2213-2262.

[38] Russo, F. and Wurzer, L. (2016). Elliptic PDEs with distributional drift and backward SDEs driven by a càdlàg martingale with random terminal time. Stoch. Dyn. Available at DOI:10.1142/S0219493717500307. Preprint HAL INRIA 01023176. 
[39] Tang, S.J. and Li, X.J. (1994). Necessary conditions for optimal control of stochastic systems with random jumps. SIAM J. Control Optim. 32 1447-1475. MR1288257

[40] Xia, J. (2000). Backward stochastic differential equation with random measures. Acta Math. Appl. Sin. Engl. Ser. 16 225-234. MR1779016

Received December 2015 and revised December 2016 